\title{
CD45 regulates GM-CSF, retinoic acid and T-cell homing in intestinal inflammation
}

\author{
A Samarakoon ${ }^{1}$, YA Shim ${ }^{1}$, M Dosanjh $^{1}$, M Crickmer $^{1}$, C Labonté-Raymond ${ }^{1}$, AA Arif ${ }^{1}$ and P Johnson ${ }^{1}$
}

CD45 is a leukocyte-specific tyrosine phosphatase important for T-cell development, and as a result, CD45 ${ }^{-1-}$ mice have substantially reduced numbers of T cells. Here we show that, upon dextran sodium sulfate (DSS)-induced colitis, CD45 ${ }^{-1-}$ mice have equivalent intestinal pathology and T-cell numbers in their colon as C57BL/6 mice and show enhanced weight loss. CD45 ${ }^{-1-}$ mice have a greater percentage of $\alpha 4 \beta 7^{+} \mathrm{T}$ cells prior to and after colitis and an increased percentage of T cells producing inflammatory cytokines in the inflamed colon, suggesting that CD45 ${ }^{-\prime-}$ effector T cells preferentially home to the intestine. In DSS-induced colitis in CD45RAG ${ }^{-{ }^{-}}$mice lacking an adaptive immune system, CD45 was required for optimal granulocyte-macrophage colony-stimulating factor (GM-CSF) and retinoic acid (RA) production by innate immune cells. Addition of $\mathrm{CD} 45^{+/+}$Tcells led to greater weight loss in the $\mathrm{RAG}^{-/-}$ mice compared with CD45RAG ${ }^{-1-}$ mice that correlated with reduced $\alpha 4 \beta 7^{+}$Tcells and lower recruitment to the colon of CD45RAG ${ }^{-1-}$ mice in DSS-induced colitis. Addition of exogenous GM-CSF to CD45RAG ${ }^{-1-}$ mice rescued RA production, increased colonic T-cell numbers, and increased weight loss. This demonstrates opposing effects of CD45 in innate and adaptive immune cells in proinflammatory responses and the expression of the gut-homing molecule, $\alpha 4 \beta 7$.

\section{INTRODUCTION}

CD45 is well established as a Src family kinase phosphatase in lymphocytes; however, the role of CD45 on myeloid cells is less well defined and its role on innate lymphoid cells (ILCs) is not known. There have been studies on in vitro-derived mast cells and dendritic cells (DCs) that have implicated CD45 as a Janusactivated kinase phosphatase regulating cytokine signaling and as a regulator of Toll-like receptor signaling, respectively. ${ }^{1-3}$ However, very few studies have examined the function of CD 45 in the intestine and the function of CD45 on immune cells during an inflammatory response has not been studied.

Three CD45-deficient mouse lines have been generated targeting exon $6\left(\mathrm{CD} 45 \mathrm{E} 6^{-1-}\right)$, exon $9\left(\mathrm{CD} 45 \mathrm{E} 9^{-1-}\right)$, and exon $12 .^{4-6}$ In this report, we characterize the $\mathrm{CD} 45 \mathrm{E}^{-/-}$and the $\mathrm{CD} 45 \mathrm{E} 9^{-1-}$ mice and also cross these mice with $\mathrm{RAG} 1^{-1-}$ mice to characterize the $\mathrm{CD} 45^{-1-}$ innate immune system in the absence of the adaptive immune system. CD45E6 ${ }^{-1-}$ have significantly reduced numbers of $\mathrm{T}$ cells in the spleen and periphery and $\mathrm{CD} 45 \mathrm{E} 9^{-1-}$ mice have even less $\mathrm{T}$ cells compared with wild-type (WT) mice., ${ }^{4,5,7}$ Although most leukocytes are negative for $\mathrm{CD} 45$, in the $\mathrm{CD} 45 \mathrm{E} 6^{-1-}$ mice, a small percentage of $\mathrm{T}$ cells express low levels of the CD45RB isoform, whereas in $\mathrm{CD} 45 \mathrm{E}^{-1-}$ mice all the leukocytes, including the T cells, are CD45 null. The CD45E6 ${ }^{-1-} \mathrm{T}$ cells express higher levels of LFA- 1 and CD $44,{ }^{8}$ and we have found increased levels of LFA-1 on CD45E ${ }^{-1-}$ T cells (unpublished data). $\mathrm{T}$ cells in the large intestine of $\mathrm{CD} 45 \mathrm{E}^{-1-}$ mice expressed more CD25 and after in vitro stimulation produced more Thelper type 1 (Th1) and Th2 cytokines than WT T cells. ${ }^{9}$ Together this suggests that $\mathrm{CD} 45^{-1-} \mathrm{T}$ cells have an activated phenotype, despite their low numbers.

Dysregulation of cytokine production by intestinal immune cells can lead to inflammatory bowel diseases. Animal models of inflammatory bowel diseases such as the dextran sodium sulfate (DSS) model have been critical in defining the role of immune cells and their cytokines in regulating inflammation in inflammatory bowel diseases. Although the DSS model of colitis is commonly used to elucidate functions of innate immune cells in the intestine upon inflammation, ${ }^{10}$ it is used less to study T cells in the intestine as DSS colitis can be induced in RAG-deficient mice. ${ }^{11}$ However, commensal antigenspecific $\mathrm{T}$ cells are induced upon DSS colitis, and this model is potentially under-used for understanding the role of T cells in colitis. ${ }^{12}$

1Department of Microbiology and Immunology, University of British Columbia, Vancouver, British Columbia, Canada. Correspondence: P Johnson (pauline@mail.ubc.ca) Received 7 May 2015; accepted 3 February 2016; published online 23 March 2016. doi:10.1038/mi.2016.23 
In addition to $\mathrm{T}$ cells, intestinal myeloid cells are important in maintaining homeostasis in the gut. In steady state, macrophages secrete interleukin (IL)-10 and exert their own regulatory function as well as help maintain the regulatory T-cell (Treg) population. ${ }^{13-15}$ DCs migrate from the intestine to the mesenteric lymph nodes (mLNs) to induce Tregs. ${ }^{16-18}$ In addition, the $\mathrm{CD} 103^{+}$DC subset has been shown to secrete retinoic acid (RA) and transforming growth factor- $\beta$ to promote Treg generation. ${ }^{19,20}$ Intestinal DCs and macrophages also secrete cytokines both at steady state and upon inflammation that influence the function of ILCs in the gut. Interleukin (IL)-23 production by intestinal myeloid cells promotes the production of cytokines by ILCs, as does tumor necrosis factor (TNF)-like protein 1A, RA, and IL-1 $\beta$ production. ${ }^{21-26}$ Conversely, granulocyte-macrophage colonystimulating factor (GM-CSF) production by ILCs is important for maintaining intestinal myeloid population numbers as, in the absence of GM-CSF, there is a reduction in DCs and macrophages in the intestine, which leads to lower RA production, and in a model of oral tolerance, insufficient generation of Tregs. ${ }^{25}$ However, the consequences of GM-CSF production by ILCs are less clear during inflammation.

In this report, we describe opposing functions for CD45 on $\mathrm{T}$ cells and myeloid cells in regulating $\mathrm{T}$-cell homing to the intestine. We show that $\mathrm{CD} 45^{-1-}$ mice have an increased percentage of $\mathrm{T}$ cells in the mLNs expressing the gut-homing molecule $\alpha_{4} \beta_{7}$ and have a higher proportion of T cells in the colon producing inflammatory cytokines in a mouse model of colitis. Conversely, in the absence of adaptive immunity, CD45 on innate immune cells promotes GM-CSF production and this stimulates RA production by DCs and enhances $\mathrm{T}$-cell homing to the colon. This supports an inflammatory role for GM-CSF and RA in the intestine.

\section{RESULTS}

CD45 expression on T cells inhibits their intestinal homing and inflammatory cytokine production limiting excessive gut inflammation

To investigate how $\mathrm{CD} 45$ regulates intestinal immune responses in vivo, we induced acute colitis in $\mathrm{WT}, \mathrm{CD} 45 \mathrm{E} 6^{-1-}$, and $\mathrm{CD} 45 \mathrm{E}^{-1-}$ mice by administering 3\% DSS in the drinking water for 7 days and then providing normal drinking water for an additional 2 days. We hypothesized that colitis would be less severe in the $\mathrm{CD} 45^{-1-}$ mice because they would have insufficient effector $\mathrm{T}$ cells. Contrary to our hypothesis, both CD45E6 $6^{-1-}$ and CD45E9 ${ }^{-1-}$ mice had significantly more weight loss than WT mice by day 9 after DSS treatment (Figure 1a) and CD45E6 ${ }^{-1-}$ mice also took longer to recover (see Supplementary Figure S1 online). Both CD45E6 $6^{-1-}$ and E9 ${ }^{-1-}$ mice had shorter colons than WT mice on day 9 of DSS colitis, indicative of enhanced intestinal inflammation, but the histology scores were comparable (Figure $\mathbf{1 b}, \mathbf{c}$ ).

Prior to colitis, as previously reported, control CD45E6 $6^{-1-}$ and $\mathrm{CD} 45 \mathrm{E} 9^{-1-}$ mice have a significantly reduced percentage of $\mathrm{T}$ cells in the spleen and mLNs compared with WT mice (Figure 1d,e). There is also a reduced percentage of $\mathrm{T}$ cells in the lamina propria (LP) of $\mathrm{CD} 45^{-1-}$ mice compared with WT mice prior to colitis (Figure 1f). Unexpectedly, there was no longer a difference in T-cell frequency in the colon of the $\mathrm{CD} 45^{-1-}$ mice after the induction of colitis, whereas there were still reduced frequencies of $\mathrm{T}$ cells in the spleen and mLNs (Figure 1d-f). This reduced frequency of $\mathrm{T}$ cells in the LP prior to colitis was also observed in the small intestine of $\mathrm{CD} 45 \mathrm{E} 6^{-1-}$ mice compared with WT mice, and this difference was also abolished with colitis (see Supplementary Figure S2). This change in T-cell frequency with colitis appeared to be specific to the small and large intestine, as reduced $\mathrm{T}$-cell frequencies were maintained in another mucosal tissue, the lung (see Supplementary Figure S2). The presence of comparable T-cell frequencies and numbers in the intestine upon colitis was unexpected as the frequencies were consistently lower prior to colitis and remained lower in other tissues. This suggested a disproportionate recruitment of $\mathrm{CD} 45^{-/-} \mathrm{T}$ cells to the inflamed colon.

To determine why there were equivalent frequencies and numbers of T cells in the LP after DSS colitis in the CD45 ${ }^{-1-}$ mice compared with WT mice, we looked at the expression of the gut-homing molecule, $\alpha_{4} \beta_{7},{ }^{27,28}$ as the loss of CD45 had previously been implicated in enhancing CD44- and integrinmediated adhesion events. ${ }^{29,30}$ We focused on the CD45E6 ${ }^{-1-}$ mice as they showed the more robust phenotype, but key findings were also reproduced in the $\mathrm{CD} 45 \mathrm{E} 9^{-1-}$ mice, indicated as data not shown. The frequency of $\alpha_{4} \beta_{7}^{+} \mathrm{T}$ cells from the mLNs of CD45E6 ${ }^{-l-}$ mice was increased both prior to and after colitis, although this did not translate into increased numbers (Figure 2a,b). This suggested that an increased percentage of gut-homing T cells in the mLNs of CD45E6 ${ }^{-1-}$ mice may help account for the preferential recruitment of $\mathrm{CD} 45^{-1-} \mathrm{T}$ cells to the intestine after colitis.

Analysis of the T cells present in the LP revealed a greater percentage of interferon- $\gamma$ (IFN $\gamma$ ), IL-17A, and GM-CSF producing $\mathrm{T}$ cells in the colon of CD45E6 $6^{-1-}$ mice on day 9 of DSS colitis compared with WT mice (Figure $\mathbf{2 c - f}$ and similarly for $\mathrm{CD} 45 \mathrm{E} 9^{-l-}$ mice, data not shown). There were no differences in the percentage or number of CD $45^{-1-}$ Foxp3 ${ }^{+}$ $\mathrm{T}$ cells in the colon of CD45E6 ${ }^{-1-}$ mice either prior to or after colitis (see Supplementary Figure S3a-c), indicating that the increased cytokine production is not due to reduced Tregs in the $\mathrm{LP}$ of $\mathrm{CD} 45^{-1-}$ mice. These results indicate that $\mathrm{CD} 45^{-1}$ - $\mathrm{T}$ cells have a greater gut-homing potential and are more proinflammatory in DSS-induced colitis.

To assess whether the $\mathrm{CD} 45^{-1-}$ innate immune system is also more proinflammatory upon DSS treatment, we next evaluated proinflammatory cytokine production from ILCs from the colon. Although there was no difference in the frequency and number of Lineage ${ }^{-}$Thy $1.2^{+}$or Lineage ${ }^{-}$ Thy $1.2^{+} \mathrm{CD} 127^{+}$cells (data not shown) in the colon between CD45E6 $6^{-1-}$ and WT mice, CD45 ${ }^{-1-}$ ILCs had an increased percentage and number of IFN $\gamma$-producing cells (Figure 3a-c), as was observed for $\mathrm{CD} 45^{-1-} \mathrm{T}$ cells in the colon. This was also seen in the $\mathrm{CD} 45 \mathrm{E} 9^{-1-}$ mice (data not shown). No differences 

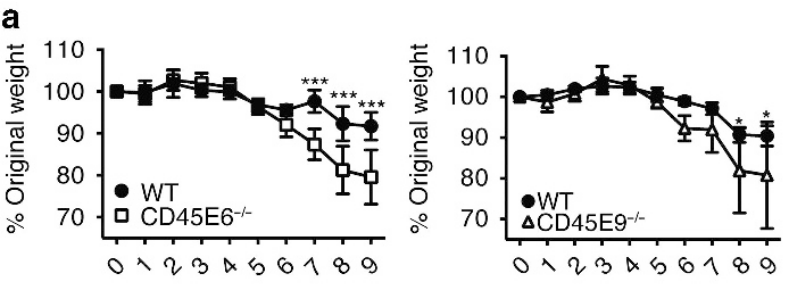

C
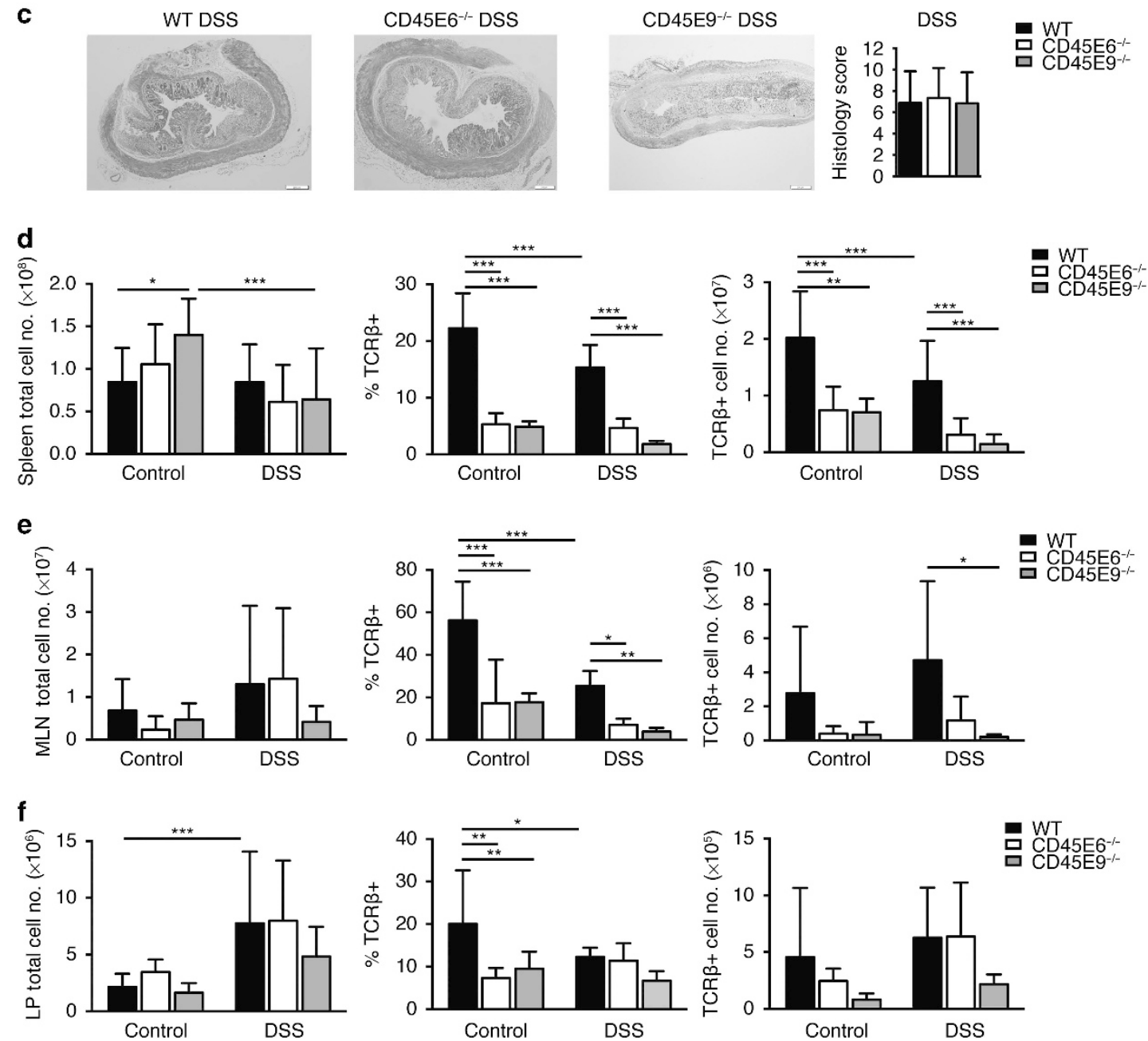

Figure 1 Characterization of wild-type (WT) and CD45 ${ }^{-1}$ - mice with dextran sodium sulfate (DSS)-induced colitis. (a) Mice were given $3 \%$ (w/v) DSS in their drinking water for 0-7 days and then water for an additional 2 days. Representative experiment is shown of the percentage of loss of body weight, repeated 7-10 times with at least 3-5 mice per group (Two-way analysis of variance (ANOVA) with repeated measures). (b) Colon length of control mice and mice on day 9 of DSS colitis. (c) Representative histology colon sections stained with hematoxylin and eosin on day 9 after colitis induction. Images are shown at $4 \times$ magnification and scale bar is $200 \mu \mathrm{m}$. Histology scores are represented in the graph on the right. Data are pooled from at least three experiments, $n=12-24$ mice per genotype. (d) Total cell number and T-cell frequency and number in the spleen of control and day 9 after colitis for WT, CD45E6 ${ }^{-1-}$, and CD45E9 ${ }^{-1}$ mice. Data are pooled from at least two experiments, $n=6-27$ mice per genotype. (e) Total cell number and T-cell frequency and number in the mesenteric lymph nodes $(\mathrm{mLNs})$ of control and mice on day 9 of DSS colitis. Data are pooled from at least two experiments, $n=6-15$ mice per genotype. (f) Total cell number and T-cell frequency and number in the colon lamina propria (LP) of control and mice on day 9 of DSS colitis. Data are pooled from at least two experiments, $n=6-24$ mice per genotype. Data represent mean \pm s.d. $P$-values were calculated with one- or two-way ANOVA; ${ }^{\star} P<0.05,{ }^{* \star} P<0.01,{ }^{* \star *} P<0.001$. TCR $\beta$, T-cell receptor $\beta$. A full color version of this figure is available at the Mucosal Immunology journal online.

were found in IL-22 (data not shown). This was not attributed to an increase in the $\mathrm{NKp} 46^{+}$ILC population and was only seen upon DSS-induced colitis, as control mice had minimal numbers of IFN $\gamma^{+}, \mathrm{IL}^{-17 \mathrm{~A}^{+}}$, or $\mathrm{GMCSF}^{+}$ILCs in the colon of WT and CD45E6 ${ }^{-1-}$ mice (data not shown). Unlike $\mathrm{CD} 45 \mathrm{E}^{-I-} \mathrm{T}$ cells however, there was no difference in the percentage or number of $\mathrm{IL}^{-17 \mathrm{~A}^{+}}$or $\mathrm{GM}^{-\mathrm{CSF}}{ }^{+}$ Lineage ${ }^{-}$Thy $1.2^{+}$cells in the colon of $\mathrm{CD} 45 \mathrm{E} 6^{-1-}$ mice compared with WT mice on day 9 of DSS treatment (Figure 3a,d,e).

Despite the enhanced GM-CSF production by CD45 ${ }^{-1-}$ $\mathrm{T}$ cells after colitis, myeloid cell frequency and number in the 
a
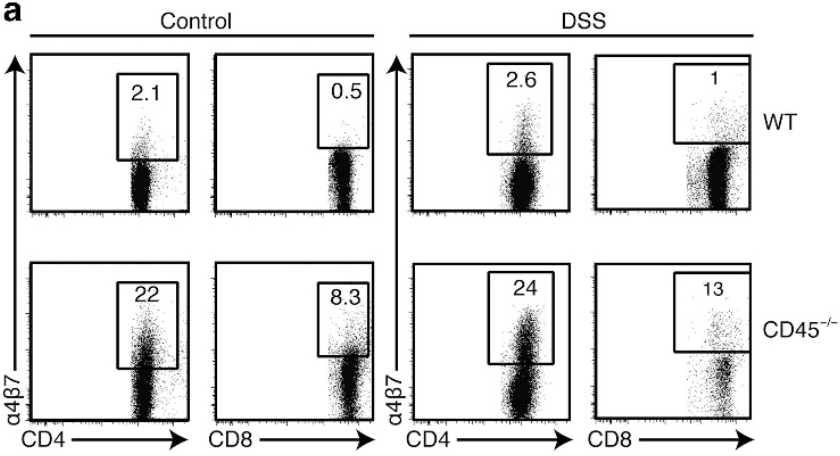

C

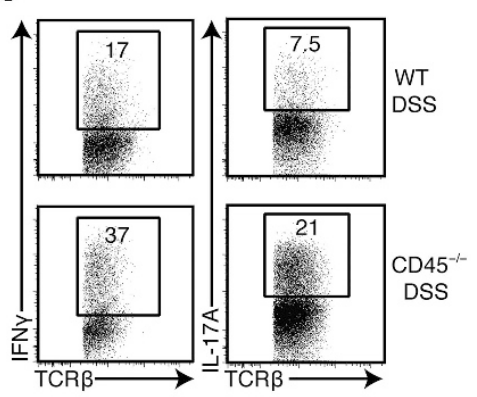

e

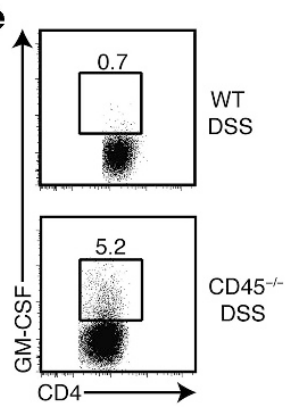

f

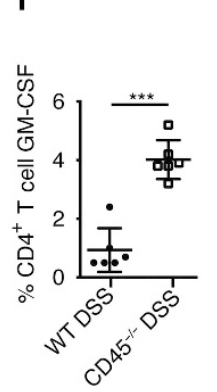

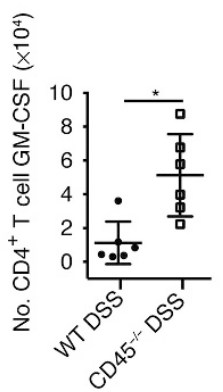

b
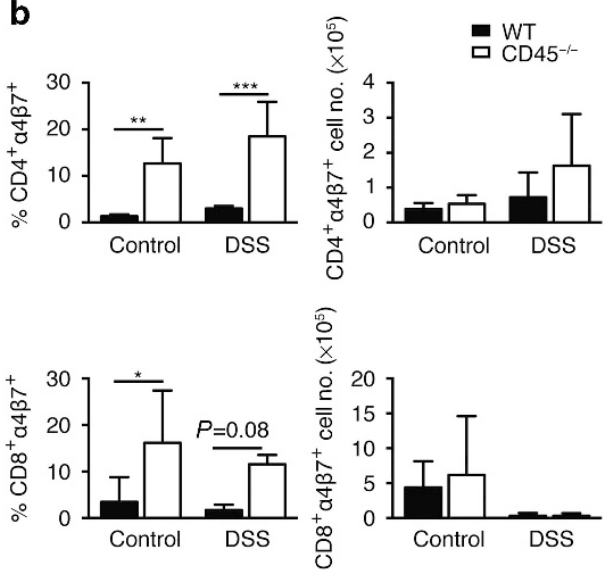

d
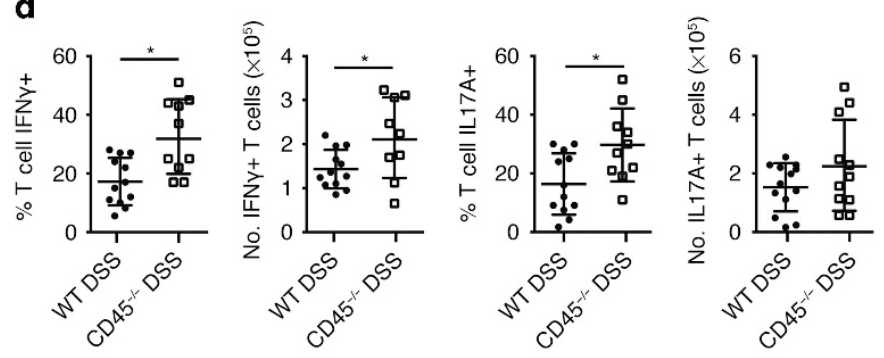

Figure $2 \mathrm{CD} 45^{-1-}$ mice have an increased percentage of gut-homing T cells in the mesenteric lymph nodes (mLNs) and enhanced proinflammatory cytokine production by colon T cells after dextran sodium sulfate (DSS). (a) Representative flow cytometry labeling of the integrin $\alpha_{4} \beta_{7}$ on CD4 ${ }^{+}$and $\mathrm{CD}^{+}{ }^{+}$cells in the $\mathrm{mLNs}$ prior to and on day 9 after DSS colitis for wild-type (WT) and CD45E6 ${ }^{-1-}$ mice. (b) Graphs of pooled data from two experiments of the frequency and cell number of $\alpha_{4} \beta_{7}^{+} \mathrm{CD} 4^{+}$or $\alpha_{4} \beta_{7}^{+} \mathrm{CD} 8^{+}$T cells, $n=6$ mice per genotype. Data represent mean $\pm \mathrm{s}$.d. and one-way analysis of variance was used to calculate $P$-values. (c) Representative flow cytometry labeling of intracellular interferon- $\gamma$ (IFN $\gamma$ ) and interleukin (IL)-17A of colon T cells from WT and CD45E6 ${ }^{-1-}$ mice after stimulation for $4-5 \mathrm{~h}$ with phorbol myristate acetate and ionomycin and treated concurrently with Brefeldin $\mathrm{A}$ on day 9 after DSS colitis. (d) Graphs of pooled data from three experiments of the frequency and cell number of IFN $\gamma^{+}$and IL-17A ${ }^{+}$colon T cells from WT and CD45E $6^{-1-}$ mice on day 9 after colitis, $n=10-12$ mice per genotype. (e) Flow cytometry of granulocyte-macrophage colony-stimulating factor (GM-CSF) production from CD4 ${ }^{+}$colon T cells on day 9 after DSS from WT and CD45E6 ${ }^{-1}$ mice. (f) Graphs of pooled data from two experiments of $\mathrm{GM}^{-\mathrm{CSF}^{+}} \mathrm{CD}^{+}{ }^{+} \mathrm{T}$ cells in the colon, $n=6$ mice per genotype. Data represent mean \pm s.d. Student's $t$-test was used for comparison between WT and CD45E6 ${ }^{-1-}$ mice in $\mathbf{d}$ and $\mathbf{f} ;{ }^{\star} P<0.05,{ }^{\star *} P<0.01,{ }^{\star \star \star} P<0.001$. TCR $\beta$, T-cell receptor $\beta$.

colons of CD45E6 ${ }^{-1-}$ mice were comparable to WT mice (Figure 3f,g). However, the percentage and number of $\mathrm{TNF} \alpha^{+}$cells within the CD11 $\mathrm{c}^{\text {hi }} \mathrm{MHCII}{ }^{\text {hi }}$ population were significantly increased in CD45E6 ${ }^{-1-}$ mice compared with WT mice upon DSS colitis (Figure 3f,h) and this was also observed in the CD45E $9^{-1-}$ mice (data not shown).

Thus CD45 negatively regulates the expression of the guthoming molecule, $\alpha_{4} \beta_{7}$, on T cells and their ability to produce IFN $\gamma$ and GM-CSF upon DSS-induced intestinal inflammation. CD45 also negatively regulates IFN $\gamma$ production by ILCs and TNF $\alpha$ by CD11 $\mathrm{c}^{\text {hi }}$ MHCII ${ }^{\text {hi }}$ myeloid cells in DSS-induced colitis.

\section{In the absence of $\mathrm{T}$ cells, there is a reduced percentage of CD45 $^{-I-}$ NK1.1 ${ }^{+}$ILCs producing IFN $\gamma$, IL-22, and GM-CSF in DSS-induced colitis}

To investigate whether the $\mathrm{CD} 45^{-1-} \mathrm{T}$ cells in the colon were responding to the hyperactive innate immune system or whether the $\mathrm{CD} 45^{-1-} \mathrm{T}$ cells were intrinsically defective, we generated CD45RAG ${ }^{-/-}$mice and induced DSS colitis. 


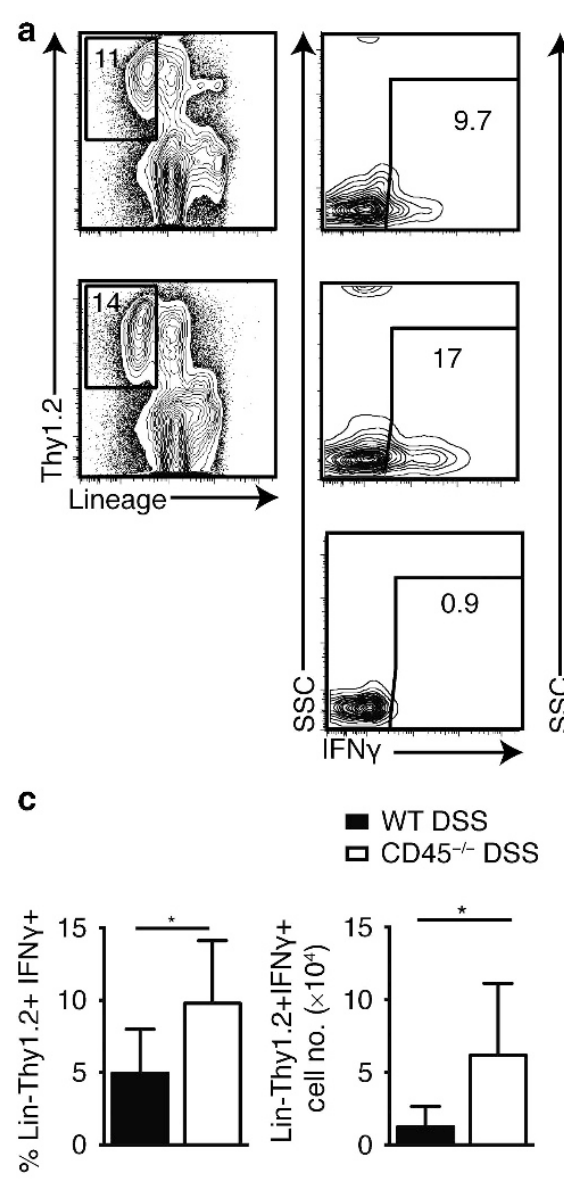

f

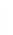

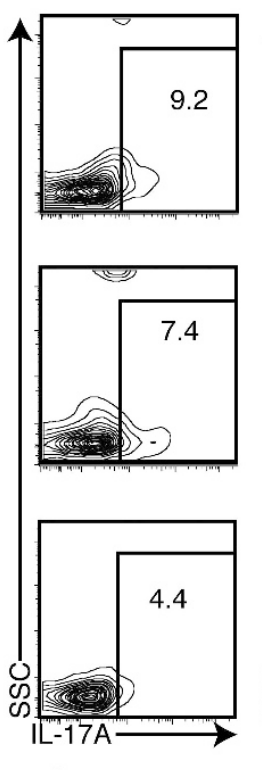

d

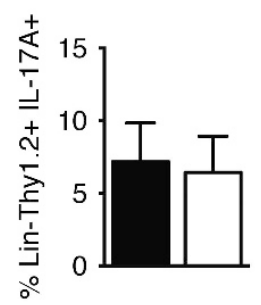

g

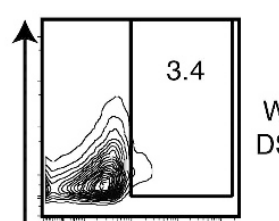

DSS

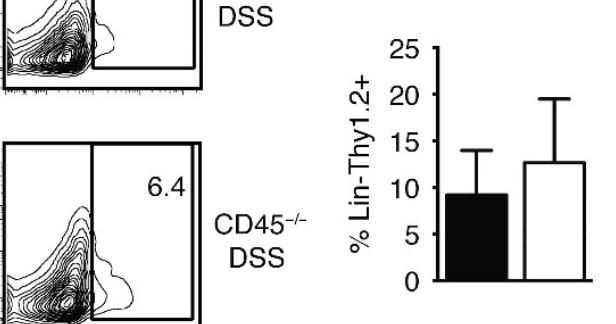

- WT DSS

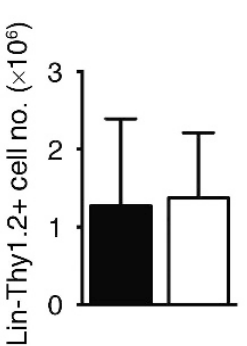

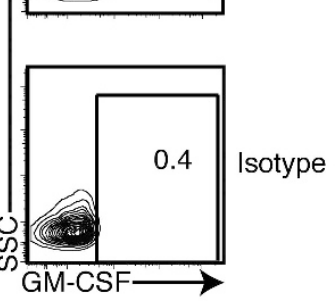

e

WT DSS

ㅁ CD45 $5^{-/-}$DSS

WT DSS

ㅁ $\mathrm{CD} 45^{-/-}$DSS
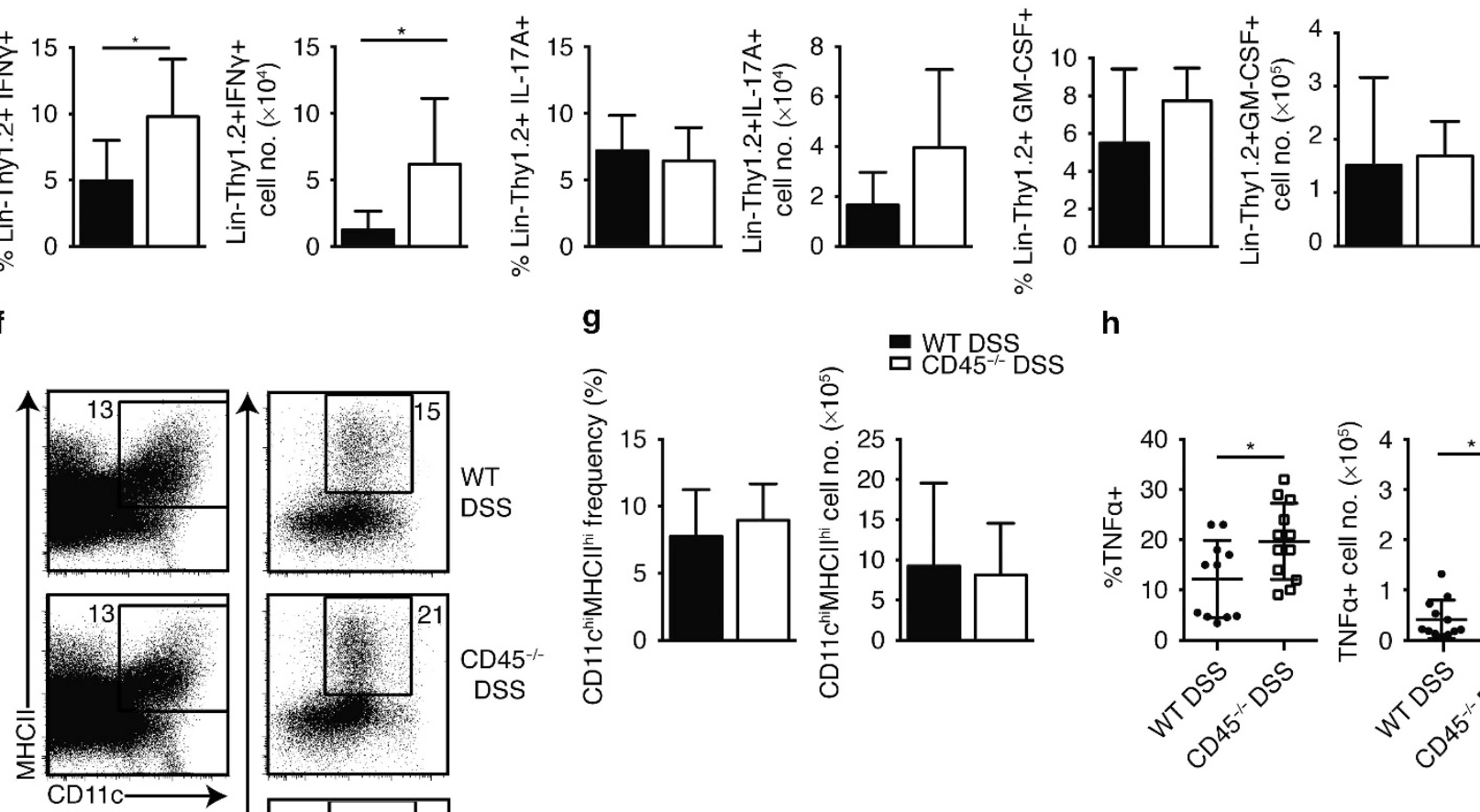

h
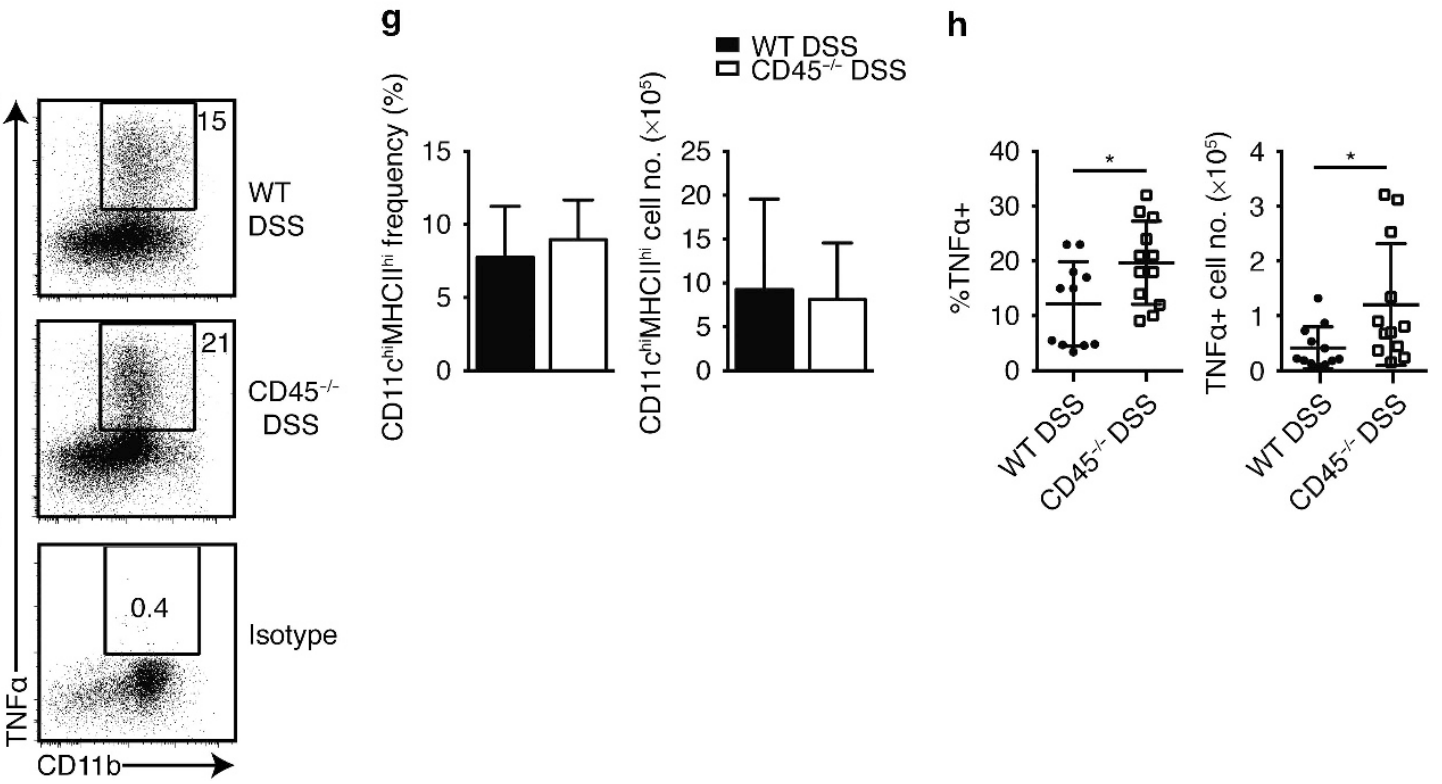

Figure $3 \mathrm{CD}^{-1-}$ mice have enhanced interferon- $\gamma(\mathrm{IFN} \gamma)$ production from Lin ${ }^{-}$Thy $1.2^{+}$innate lymphoid cells and tumor necrosis factor- $\alpha$ (TNF $\left.\alpha\right)$ production from $\mathrm{CD} 11 \mathrm{c}^{\mathrm{hi}} \mathrm{MHCII}{ }^{\text {hi }}$ myeloid cells in the colon after colitis. (a) Representative flow cytometry labeling of colon Lineage ${ }^{-}$Thy $1.2^{+}$and their production of IFN $\gamma$, interleukin (IL)-17A and granulocyte-macrophage colony-stimulating factor (GM-CSF) and their respective isotype controls from wildtype (WT) and CD45E6 ${ }^{-1-}$ mice after stimulation for 4-5 h with phorbol myristate acetate and ionomycin and treated concurrently with Brefeldin A on day 9 after dextran sodium sulfate (DSS). Pooled data from at least two experiments of Lin ${ }^{-}$Thy $1.2^{+}$cell frequency and number (b) in the colon and the percentage and number of (c) IFN $\gamma^{+}$, (d) IL-17A ${ }^{+}$, and (e) GM-CSF ${ }^{+}$cells within that population, $n=6-13$ mice per genotype. (f) Representative flow

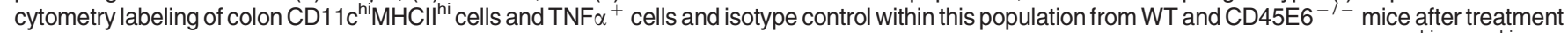
with Brefeldin A for 4-5 h on day 9 after DSS. Graphs are pooled data from three experiments of $(\mathbf{g})$ the frequency and cell number of CD11 $\mathrm{c}^{\text {hi }} \mathrm{MHC} \mathrm{II}^{\mathrm{hi}} \mathrm{cells}$ and (h) the percentage and number of TNF $\alpha^{+}$cells within this population, $n=11-12$ mice per genotype. Data represent mean \pm s.d. Student's $t$-test was used for comparison between WT and CD45E6 ${ }^{-1-}$ mice; ${ }^{*} P<0.05$. MHC, major histocompatibility complex; SSC, side scatter. 
Unlike the WT and $\mathrm{CD} 45^{-/-}$mice, after colitis induction there was no significant difference in weight loss between $\mathrm{RAG}^{-1-}, \mathrm{CD} 45 \mathrm{RAG}^{-1-}$ and CD45E9RAG ${ }^{-1-}$ mice (Figure 4a,b). Notably, CD45 was undetectable on innate immune cells in the CD45E6RAG ${ }^{-1-}$ mice, making them comparable to the CD45E9RAG ${ }^{-1-}$ mice. Therefore, CD45E9RAG ${ }^{-1-}$ mice (hereafter referred to as CD45RAG ${ }^{-1-}$ mice) were used for further analysis; however, we did confirm the key results in the CD45E6RAG ${ }^{-1-}$ mice. The CD45RAG ${ }^{-1-}$ mice had shorter colons than the RAG ${ }^{-1-}$ mice and enhanced intestinal inflammation assessed by histology but had no difference in total cell number in the colon after DSS-induced colitis (Figure $4 \mathbf{c}-\mathbf{e}$ ). Upon DSSinduced colitis, CD45RAG ${ }^{-1-}$ mice had comparable numbers and frequencies of Lin ${ }^{-}$Thy $1.2^{+}$and Lin ${ }^{-}$Thy1.2 $2^{+} \mathrm{NK} 1.1^{+}$ ILCs to $\mathrm{RAG}^{-/-}$mice (see Supplementary Figure S4). However, the Lin $^{-}$Thy $1.2^{+}$NK1.1 $1^{+}$ILCs in the inflamed colon of CD45RAG ${ }^{-1-}$ mice had a lower percentage of cells producing IFN $\gamma$, IL-22, and GM-CSF and significantly reduced numbers of cells making GM-CSF compared with the RAG ${ }^{-1-}$ mice (Figure $\mathbf{4} \mathbf{f}-\mathbf{h}$ ). This was unexpected because there was more inflammation in the colons of CD45RAG ${ }^{-1-}$ mice, as assessed by colon length and histology. As ILC IL-22 has been shown to be protective in DSS colitis, ${ }^{24}$ one possible explanation is that the reduced IL-22 production by these cells may contribute to the enhanced gut inflammation in the CD45RAG ${ }^{-1-}$ mice. Furthermore, this suggests that it is not the hyperactivation of $\mathrm{CD} 45^{-1-}$ innate immune cells that leads to increased cytokine production by $\mathrm{CD} 45^{-1-} \mathrm{T}$ cells, rather it appears to be the opposite.

\section{CD45 helps promote RA production by DCs in the mLNs in DSS-induced colitis}

There have been several studies examining the impact of myeloid cell secretory factors on ILCs as well as the crosstalk between ILCs and myeloid cells in the gut. ${ }^{22,24,25}$ DCs are also an important source of RA in the gut, and RA promotes IL-22 production by ILCs thereby attenuating inflammation in DSS colitis. ${ }^{22}$ Therefore, we wanted to assess whether the reduction in cytokine production by the NK1.1 $1^{+}$ILCs in the CD45RAG ${ }^{-1-}$ mice upon DSS treatment could be due to alterations in myeloid cells. CD45RAG ${ }^{-1-}$ mice consistently had a reduced frequency of $\mathrm{CD} 11 \mathrm{c}^{\mathrm{hi}} \mathrm{MHCII}{ }^{\text {hi }}$ cells in the colon of DSS-treated mice (Figure 5a). However, unlike CD45 ${ }^{-1-}$ mice, there was no increase in TNF $\alpha$ production from CD11c ${ }^{\text {hi }}$ MHCII $^{\text {hi }}$ cells in the colon after DSS compared with the $\mathrm{RAG}^{-1-}$ mice (Figure 5a). Therefore, we evaluated RA production capacity by DCs after DSS treatment using ALDEFLUOR, a synthetic substrate that accumulates in cells after cleavage and is proportional to active aldehyde dehydrogenases, the enzymes involved in RA production. In control mice, there was a trend for less ALDEFLUOR-positive CD11 $\mathrm{c}^{\text {hi }}$ MHCII ${ }^{\text {hi }}$ cells in the colon of CD45RAG ${ }^{-1-}$ mice compared with $\mathrm{RAG}^{-1-}$ mice, and this became a significant difference in cell number on day 9 of DSS colitis (see Supplementary Figure S5). A similar trend was observed in DCs in the mLNs of CD45RAG ${ }^{-1-}$ after DSS treatment, with a significant drop in the percentage of ALDEFLUOR-positive DCs, the majority of which expressed CD103 (Figure 5b). Interestingly, there was also a reduced percentage of ALDEFLUOR-positive DCs in the mLNs before and after DSS-treatment in CD45 ${ }^{-1-}$ mice, but these differences were not significant in DCs from the colon of these $\mathrm{CD} 45^{-1-}$ mice (see Supplementary Figure S6). These results suggest a role for $\mathrm{CD} 45$ in promoting RA production by DCs in the mLNs in DSS-induced colitis.

The absence of CD45 on the innate immune system delays CD45 ${ }^{+I+}$ T-cell homing and attenuates colitis

DSS-induced colitis in the $\mathrm{CD} 45^{-1-}$ mice resulted in an increased percentage of $\mathrm{T}$ cells in the colon producing the proinflammatory cytokines (IFN $\gamma$ and GM-CSF), increased IFN $\gamma$ production by ILCs, and increased TNF $\alpha$ by DCs. In contrast, DSS-induced colitis in CD45RAG ${ }^{-1-}$ mice did not show a similar increase in IFN $\gamma$ production by ILCs or increased TNF $\alpha$ by DCs; in fact, there were reduced percentages of IFN $\gamma$, GM-CSF, and IL-22 producing NK1.1 ${ }^{+}$ILCs in the colon of these mice. This suggested that adaptive immune cells such as T cells may crosstalk with the innate immune cells to promote cytokine production by ILCs and myeloid cells. To assess the effect of the CD45 $5^{-/-}$innate immune cells on normal T cells, we adoptively transferred polyclonal $\mathrm{CD} 45^{+/+} \mathrm{T}$ cells from naive WT mice into $\mathrm{RAG}^{-1-}$ and CD45RAG ${ }^{-1-}$ mice and then induced DSS colitis. Although the addition of T cells enhanced the weight loss in the $\mathrm{RAG}^{-1-}$ mice, there was a significant delay in weight loss in the CD45RAG ${ }^{-1-}$ mice (Figure 6a). Consistent with this delay in systemic inflammation, CD45RAG ${ }^{-1-}$ mice had longer colons and reduced cell numbers in the LP on day 8 after DSS treatment, suggesting lower intestinal inflammation in these mice compared with $\mathrm{RAG}^{-1-}$ mice (Figure $\mathbf{6 b}, \mathbf{c}$ ). This was in contrast to the $\mathrm{CD} 45^{-1-}$ mice, which showed greater weight loss compared with WT mice in DSS-induced colitis, raising the possibility that the $\mathrm{CD} 45^{-/-} \mathrm{T}$ cells are acting differently to $\mathrm{CD} 45^{+/+} \mathrm{T}$ cells.

The transfer of $\mathrm{CD} 45^{+/+}$polyclonal $\mathrm{T}$ cells did not restore the reduced frequency or number of CD11 $\mathrm{c}^{\mathrm{hi}} \mathrm{MHCII}{ }^{\mathrm{hi}}$ cells in the colon of $\mathrm{CD} 45 \mathrm{RAG}^{-1-}$ mice after DSS treatment (Figure 6d). Further analysis revealed that, after colitis induction, CD45RAG ${ }^{-1-}$ mice had significantly reduced frequency and numbers of both $\mathrm{CD} 4^{+}$and $\mathrm{CD} 8^{+} \mathrm{T}$ cells in the colon and mLNs (Figure 6e-h). This reduction of T cells in the gut of CD45RAG ${ }^{-1-}$ mice compared with the $\mathrm{RAG}^{-1-}$ mice may help explain the reduced severity of DSS-induced colitis in these mice. A recent study demonstrated that, upon the transfer of polyclonal CD45 ${ }^{+1+} \mathrm{T}$ cells into naive CD45RAG ${ }^{-1-}$ and $\mathrm{RAG}^{-1-}$ mice, there was reduced lymphopenia-induced proliferation of the transferred $\mathrm{T}$ cells in CD45RAG ${ }^{-1-}$ mice, resulting in lower frequency and number of CD $45^{+1+}$ $\mathrm{T}$ cells in the spleen and peripheral lymph nodes on day 7 after transfer. ${ }^{7}$ However, upon inflammation induced by DSS, there was no significant difference in T-cell frequency or number in the spleen between CD45RAG ${ }^{-/-}$and $\mathrm{RAG}^{-1-}$ 
a

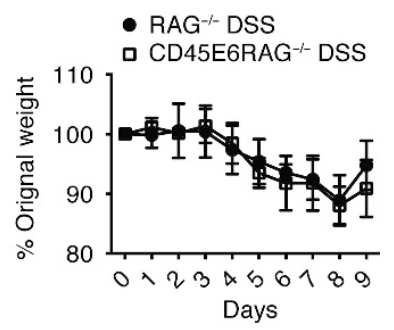

b

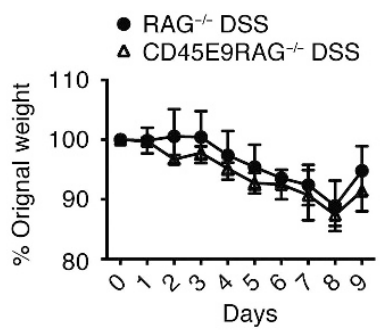

c

믈 $\mathrm{RAG}^{-/-}$

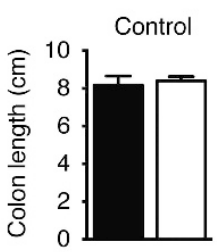

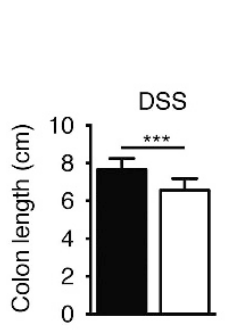

d

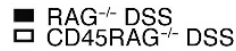

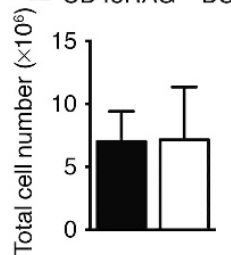

e
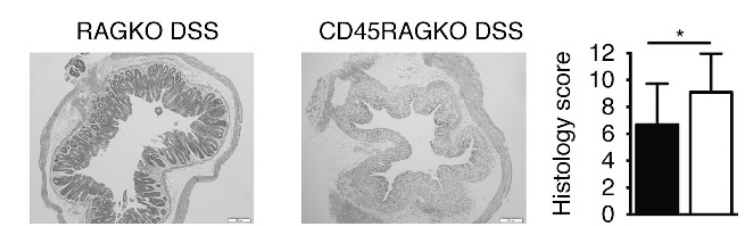

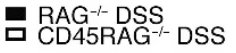

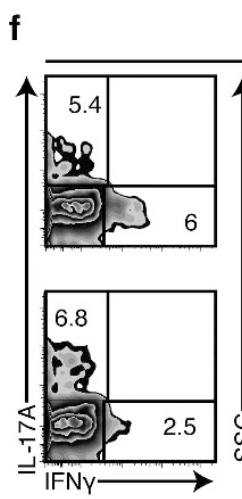

Lin-Thy1.2+
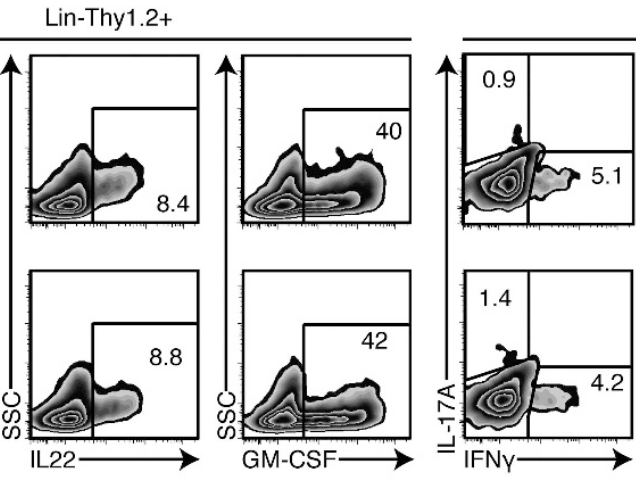

Lin-Thy1.2+NK1.1+
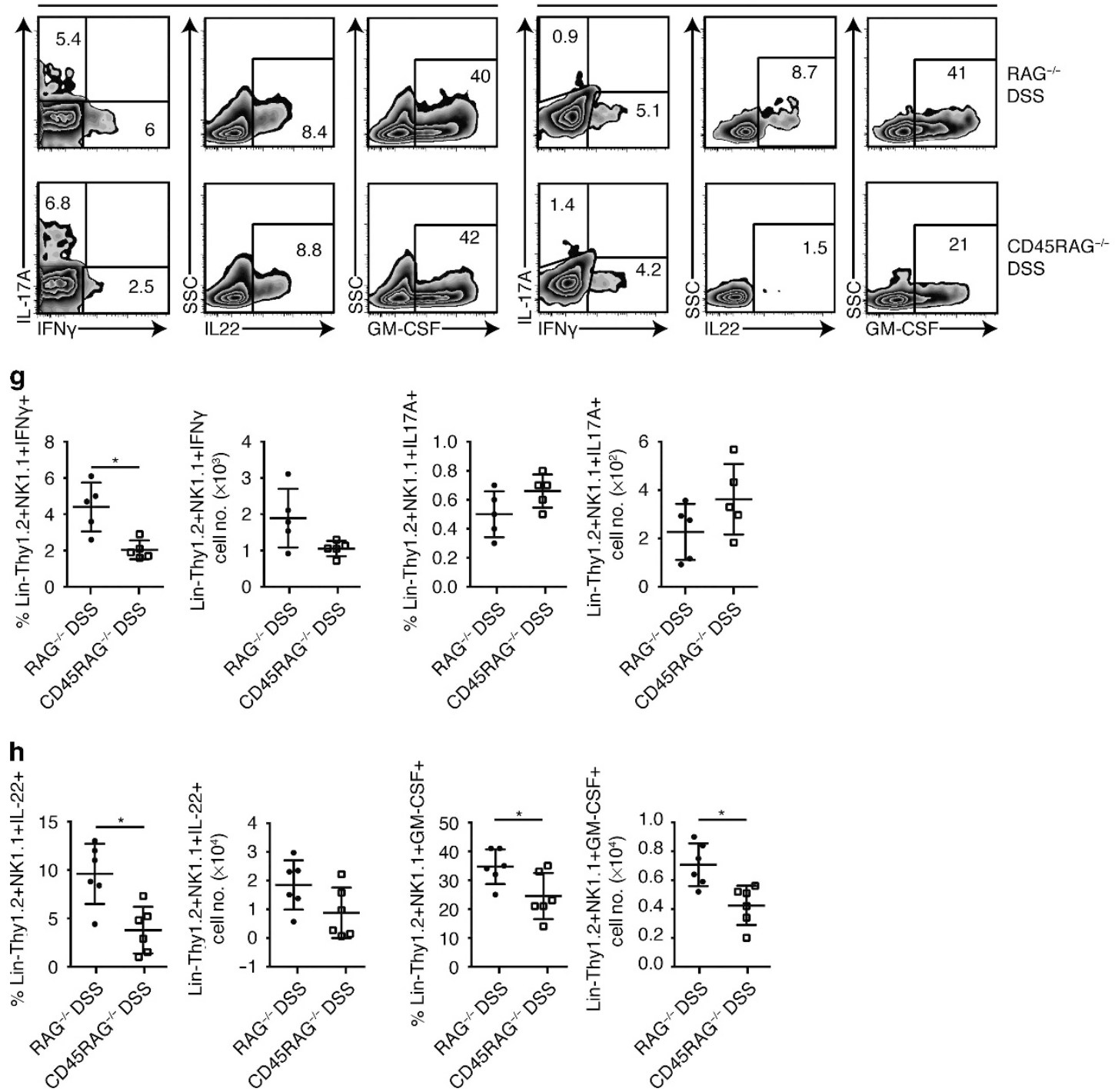
mice (see Supplementary Figure S7), suggesting that lymphopenia-induced proliferation is not a contributing factor to the reduction of colon $\mathrm{T}$ cells observed in $\mathrm{CD}_{4} \mathrm{RAG}^{-1-}$ mice. In addition, there was no significant increase in the percentage of Foxp $3^{+} \mathrm{T}$ cells within $\mathrm{CD} 4{ }^{+}$or $\mathrm{CD}^{+} \mathrm{T}$ cells in the spleen, mLNs, and $\mathrm{LP}$ of the CD45RAG ${ }^{-/-}$ mice (see Supplementary Figure S8), indicating that increased numbers of Tregs were not the reason for the reduced colitis observed in CD45RAG ${ }^{-1-}$ mice.

As there was a reduced frequency of DCs from the mLNs of $\mathrm{CD}_{4} \mathrm{RAG}^{-1-}$ mice producing RA after DSS treatment, we evaluated whether insufficient RA could be reducing $\mathrm{T}$-cell a
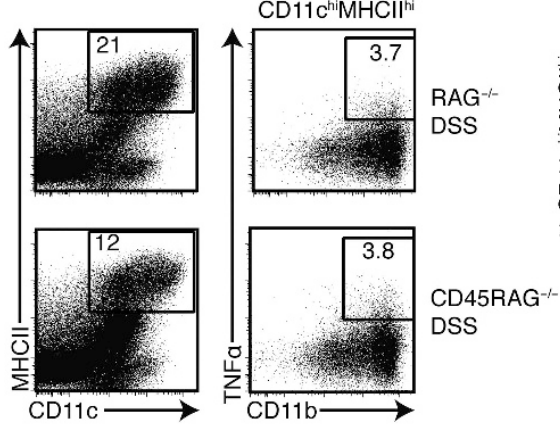

b

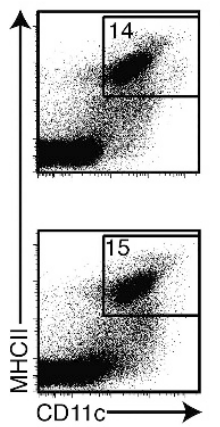

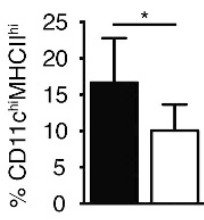

\%

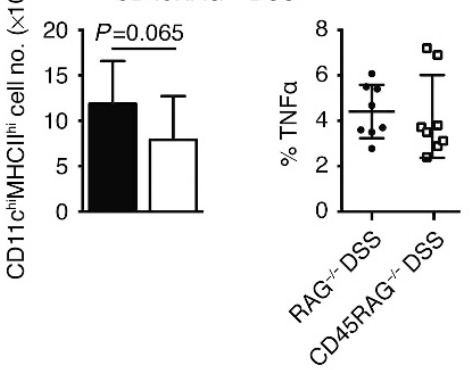

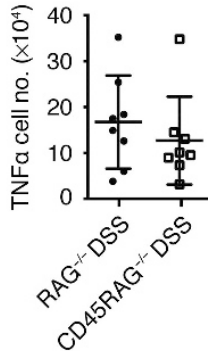
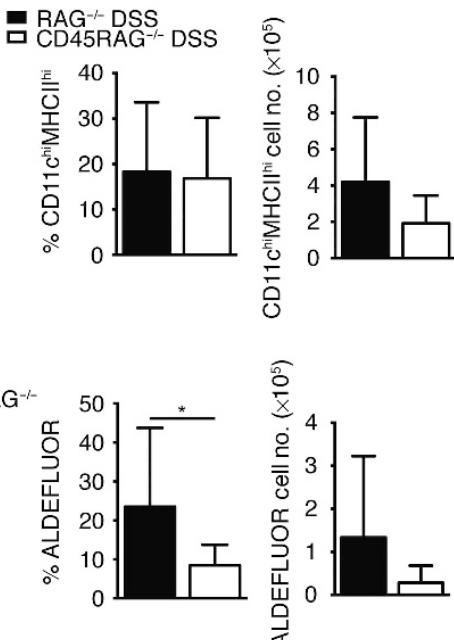

Figure $5 \mathrm{CD} 45 \mathrm{RAG}^{-1-}$ mice have reduced frequency of $\mathrm{CD} 11 \mathrm{c}^{\text {hi }} \mathrm{MHCl}$ i ${ }^{\text {hi }}$ cells in the colon and a reduced percentage of retinoic acid-producing dendritic cells (DCs) in the mesenteric lymph nodes (mLNs) in dextran sodium sulfate (DSS) colitis. (a) Flow cytometry (left) and the frequency and cell number (right) of $\mathrm{CD} 11 \mathrm{c}^{\text {hi }} \mathrm{MHCII}^{\text {hi }}$ cells and TNF $\alpha^{+}$cells within this population in the colon of RAG ${ }^{-}-$and $\mathrm{CD} 45 \mathrm{E} 9 \mathrm{RAG}{ }^{-1}$ mice on day 9 after DSS. Data are pooled from at least two experiments, $n=8-12$ mice per genotype. (b) Flow cytometry (left) of CD $11 c^{\text {hi }} \mathrm{MHCII}{ }^{\text {hi }}$ cells in the mLNs and the percentage of ALDEFLUOR ${ }^{+}$cells as well as the negative control (DEAB) for ALDEFLUOR within CD11c ${ }^{\text {hi MHCII }}{ }^{\text {hi }}$ cells after DSS. Graphs of the frequency and cell number of DCs and ALDEFLUOR ${ }^{+}$DCs in the $\mathrm{mLNs}$ after DSS (right). Data are pooled from at least two experiments and represents mean \pm s.d., $n=5-10$ mice per genotype. Student's $t$-test was used for comparison and ${ }^{\star} P<0.05$. MHC, major histocompatibility complex; TNF, tumor necrosis factor.

Figure 4 Characterization of RAG and CD45RAG ${ }^{-1-}$ mice and the reduced percentage of interferon- $\gamma$ (IFN $\gamma$ ), interleukin (IL)-22, and granulocytemacrophage colony-stimulating factor (GM-CSF) producing Lin ${ }^{-}$Thy $1.2^{+} \mathrm{NK} 1.1^{+}$innate lymphoid cells in the colon of CD45RAG ${ }^{-1-}$ mice after dextran sodium sulfate (DSS) colitis. RAG ${ }^{-1-}$, (a) CD45E6RAG ${ }^{-1-}$, and (b) CD45E9RAG ${ }^{-1-}$ mice were given $3 \%$ (w/v) DSS in their drinking water for 7 days and then water for an additional 2 days. Representative experiment of the percentage of loss of body weight is shown and was repeated $5-12$ times with at least 3-5 mice per genotype (two-way analysis of variance with repeated measures). (c) Colon lengths of untreated (left) RAG ${ }^{-1-}$ and CD45E9RAG ${ }^{-1-}$ mice and on day 9 of DSS colitis (right). (d) Pooled data from five experiments of colon lamina propria cell number of RAG ${ }^{-1-}$ and CD45E9RAG ${ }^{-1-}$ mice on day 9 after DSS, $n=17$ mice per genotype. (e) Representative histology colon sections stained with hematoxylin and eosin on day 9 after colitis induction are shown. Images are shown at $\times 4$ magnification and scale bar is $200 \mu \mathrm{m}$. (f) Representative flow cytometry labeling of IFN $\gamma$, IL-17A, IL-22, and GM-CSF from Lineage ${ }^{-}$Thy $1.2^{+}$(left) and Lineage ${ }^{-}$Thy $1.2^{+}$NK1.1 $1^{+}$(right) cells in the colon from RAG ${ }^{\prime-}$ and CD45E9RAG ${ }^{-}$ mice on day 9 after DSS. Pooled data from two experiments of the frequency and cell number of (f) IFN $\gamma^{+}$and IL-17A ${ }^{+}$as well as $(\mathbf{g}) \mathrm{IL}_{-2} 22^{+}$and $\mathrm{GM}_{-\mathrm{CSF}}{ }^{+}$cells from Lin ${ }^{-}$Thy $1.2^{+} \mathrm{NK} 1.1^{+}$cells in the colon of RAG ${ }^{-1-}$ and CD45E9RAG ${ }^{-1-}$ mice on day 9 after DSS, $n=5-6$ mice per genotype. Data represent mean \pm s.d. Student's $t$-test was used for comparison between RAG ${ }^{-1-}$ and CD45RAG ${ }^{-1-}$ mice in $\mathbf{c}-\mathbf{e}, \mathbf{g}$ and $\mathbf{h} ;{ }^{\star} P<0.05$, ${ }^{\star \star \star} P<0.001$. SSC, side scatter. A full color version of this figure is available at the Mucosal Immunology journal online. 
homing to the intestine. Although the importance of RA in Treg homing to the gut is well established, emerging evidence also suggests a role for RA in effector Th1 homing to the gut. ${ }^{31,32}$ As
RA imprints T-cell homing to the gut by upregulating $\alpha_{4} \beta_{7}$ expression on T cells, we examined $\alpha_{4} \beta_{7}$ expression on T cells in the mLNs after DSS treatment and found that the transferred a
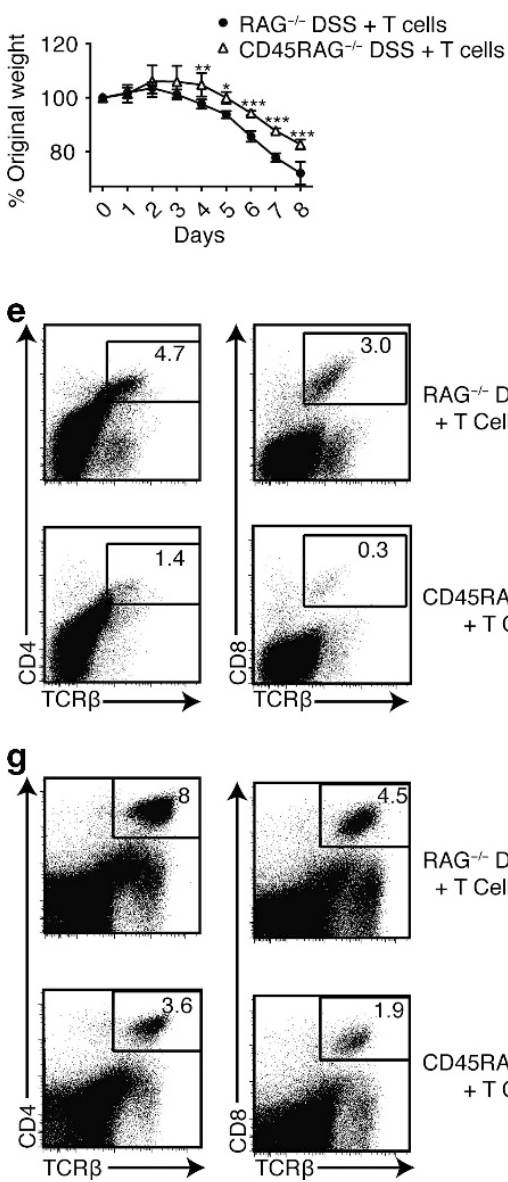

b

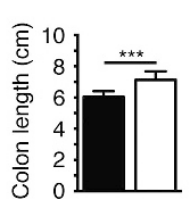

c

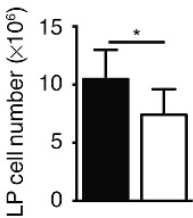

d

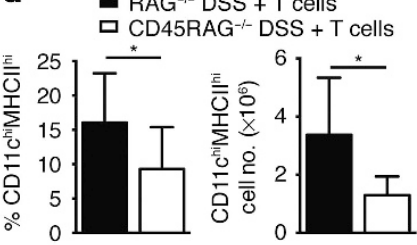

f
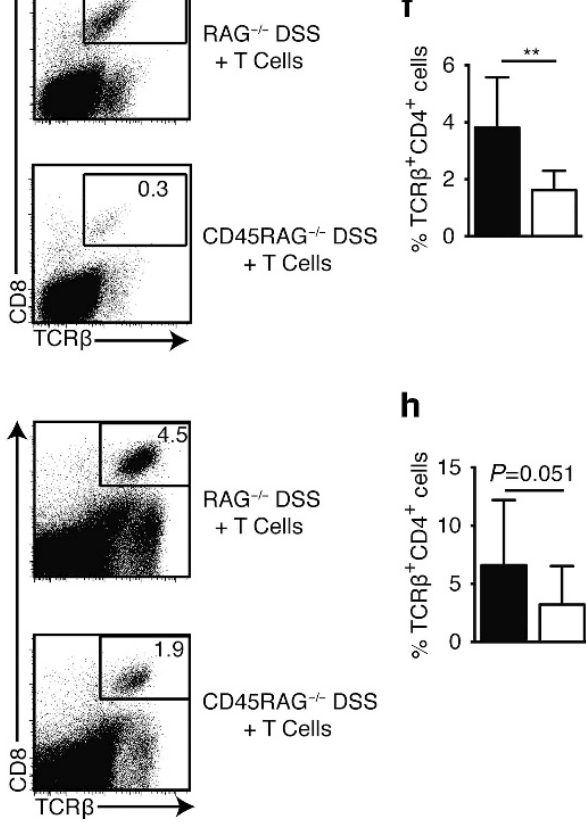

h

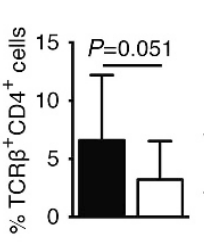

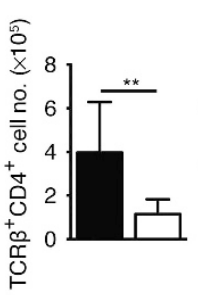
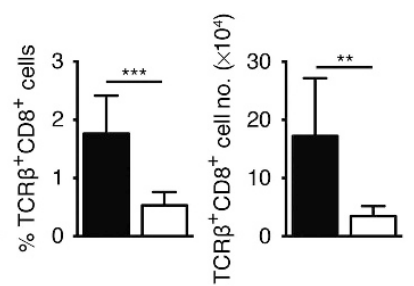

- $\mathrm{RAG}^{-1-} \mathrm{DSS}+\mathrm{T}$ cells ㅁ CD45RAG ${ }^{--}$DSS + T cells
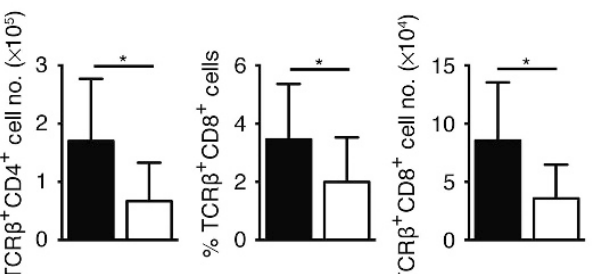

Figure 6 The transfer of CD45-sufficient wild-type (WT) polyclonal T cells reduces weight loss and T-cell infiltration in the colon of CD45RAG ${ }^{-1-}$ mice with dextran sodium sulfate (DSS)-induced colitis. (a) Mice were injected with $3-5 \times 10^{6} \mathrm{~T}$ cells intravenously on day 0 and were given $3 \%$ (w/v) DSS in their drinking water for 0-7 days and then put on water for an additional 1-2 days. Graph is representative of the percentage of loss of body weight in $\mathrm{RAG}^{-1-}$ and CD45E9RAG ${ }^{-1-}$ mice and conducted four times with at least 2-3 mice per genotype. Pooled data from at least three experiments of (b) colon length and (c) colon cell number of $\mathrm{RAG}^{-1-}$ and $\mathrm{CD} 45 \mathrm{E} 9 \mathrm{RAG}{ }^{-1-}$ mice on day 8 after DSS and T-cell injection, $n=9-14$ mice per genotype. (d) Pooled data from three experiments of colon CD11 $\mathrm{c}^{\text {hi }} \mathrm{MHCII}$ cell frequency and number on day 8 after DSS and T-cell transfer in RAG ${ }^{-/}-$and CD45E9RAG ${ }^{-1-}$ mice, $n=9-10$ mice per genotype. (e) Flow cytometry and (f) pooled data from at least three experiments of CD4 ${ }^{+}$and CD8 ${ }^{+} \mathrm{T}_{\text {cells }}$ from the colon of RAG ${ }^{-1-}$ and CD45E9RAG ${ }^{-1-}$ mice on day 8 after DSS and T-cell injection, $n=10-13$ mice per genotype. (g) Flow cytometry and (h) pooled data from at least four experiments of $\mathrm{CD} 4^{+}$and $\mathrm{CD} 8^{+} \mathrm{T}^{\text {cells }}$ from the mesenteric lymph node of RAG ${ }^{-1-}$ and $\mathrm{CD} 45 \mathrm{E9RAG}{ }^{-1-}$ mice on day 8 after DSS and T-cell injection, $n=13-16$ mice per genotype. Data represent mean \pm s.d. Two-way analysis of variance with repeated measures (a) and Student's $t$-test $(\mathbf{b}-\mathbf{d}, \mathbf{f}, \mathbf{h})$ was used for comparison between RAG ${ }^{-l-}$ and CD45E9RAG ${ }^{-1-}$ mice; ${ }^{\star} P<0.05,{ }^{* *} P<0.01,{ }^{* * *} P<0.001$. LP, lamina propria; MHC, major histocompatibility complex; TCR, T-cell receptor.

Figure 7 Exogenous retinoic acid (RA) and granulocyte-macrophage colony-stimulating factor (GM-CSF) enhance dextran sodium sulfate (DSS) colitis in CD45RAG ${ }^{-1}$ mice. (a-e) Representative flow cytometry labeling and pooled data from four experiments showing the percentage and mean fluorescence intensity (MFI) of $\alpha_{4} \beta_{7}$ and C-C motif chemokine receptor 9 (CCR9) expression on CD4 ${ }^{+}$or CD8 ${ }^{+}$T cells in the mesenteric lymph nodes (mLNs) of RAG ${ }^{-1-}$ and CD45E9RAG ${ }^{-1-}$ mice on day 8 after DSS and T-cell transfer, $n=11-13$ mice per genotype. Student's $t$-test was used for comparison from b-e. Mice were injected with $3-5 \times 10^{6} \mathrm{~T}$ cells intravenously on day 0 and were given $3 \%$ DSS (w/v) in their drinking water for $0-7$ days and then regular water for an additional 1-2 days and (f) $200 \mu \mathrm{g}$ per mouse of RA or vehicle or (g) $5 \mu \mathrm{g}$ per mouse of GM-CSF or phosphate-buffered saline (PBS) was injected intraperitoneally into mice every other day from day 1. Representative experiments from two conducted of the percentage of loss of body weight are shown, $n=3-5$ mice per genotype (two-way analysis of variance (ANOVA) with repeated measures). Pooled data from two experiments of colon length after DSS with (f) RA or vehicle or (g) GM-CSF or PBS, $n=5-6$ mice per genotype. (h) Pooled data from two experiments of the frequency and cell numbers of $\mathrm{CD}^{+}{ }^{+}$and $\mathrm{CD} 8{ }^{+} \mathrm{T}^{\mathrm{T}}$ cells in the colon after DSS + polyclonal T-cell transfer and GM-CSF or PBS injection. (i) Pooled data from two experiments of the cell frequency and number of ALDEFLUOR expression from CD $11 \mathrm{c}^{\mathrm{h}} \mathrm{MHCII}{ }^{\text {hi }} \mathrm{CD} 103^{+}$cells from the $\mathrm{mLNs}$ after DSS + polyclonal $\mathrm{T}$-cell transfer and with or without GM-CSF injection. Data are represented as mean \pm s.d. One- and two-way ANOVA was used for comparison from $\mathbf{f}-\mathrm{i}$ and ${ }^{\star} P<0.05,{ }^{* \star} P<0.01,{ }^{* \star \star} P<0.001$. TCR, T-cell receptor. 
a

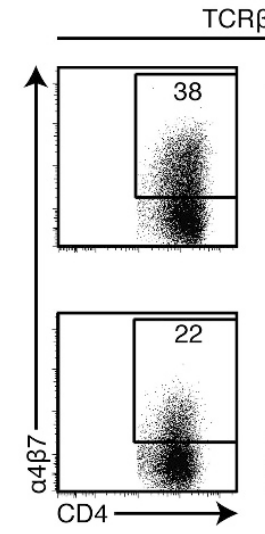

$\mathrm{TCR} \beta^{+} \mathrm{CD} 4^{+}$
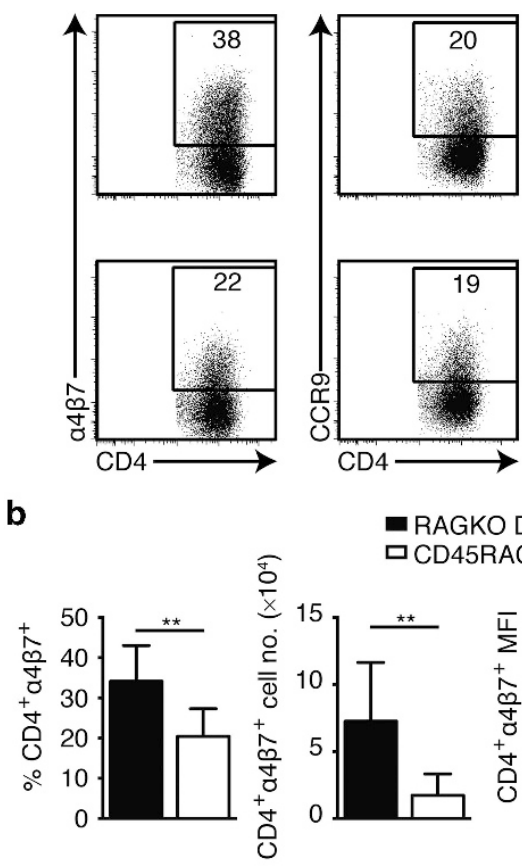

d
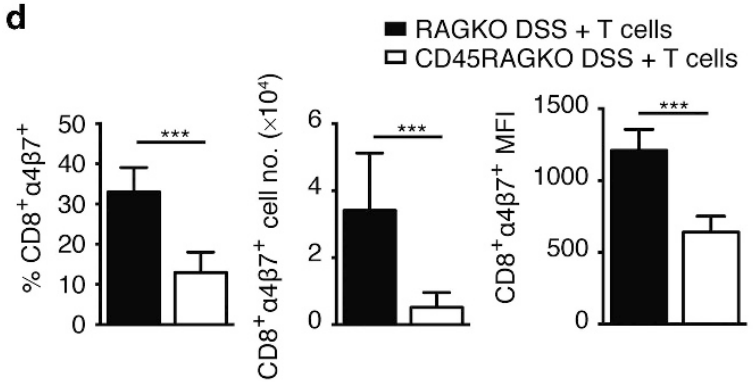

f

- RAGKO DSS + T cells + Vehicle a CD45RAGKO DSS + T cells + Vehicle

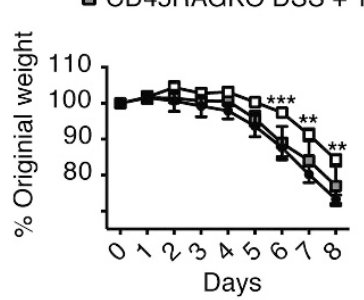

h

- RAGKO DSS + T cells + PBS

- CD45RAGKO DSS + T cells + PBS

- CD45RAGKO DSS + T cells + GM-CSF

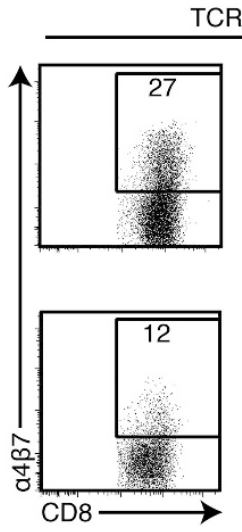

$\mathrm{TCR}^{+} \mathrm{CD}^{+}$

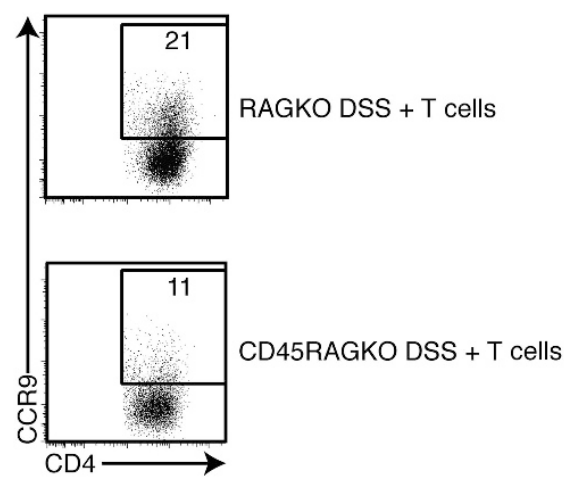

c

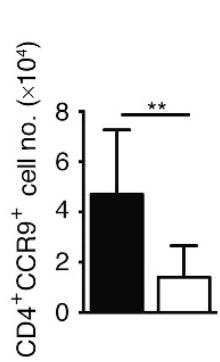

- RAGKO DSS + T cells 口 CD45RAGKO DSS + T cells

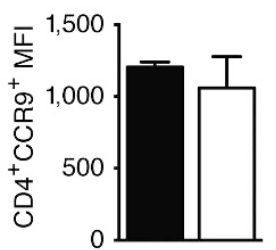

e

- RAGKO DSS + T cells ㅁ CD45RAGKO DSS + T cells
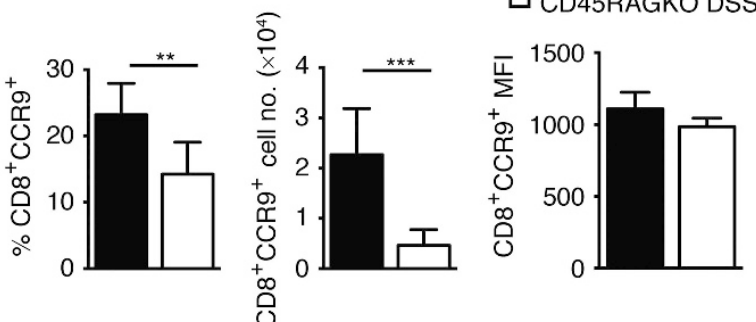

\section{g}

- RAGKO DSS + T cells + PBS

a CD45RAGKO DSS + T cells + PBS

a CD45RAGKO DSS + T cells + GM-CSF
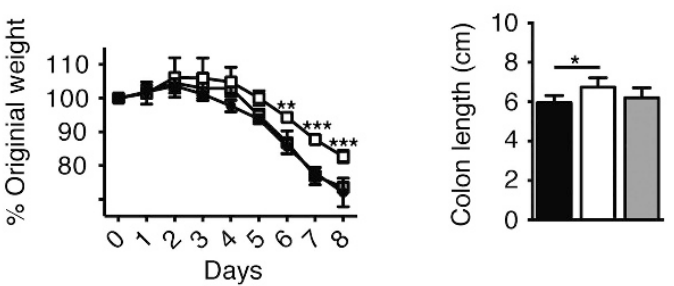

- RAGKO DSS + T cells + PBS

口 CD45RAGKO DSS + T cells + PBS

口 CD45RAGKO DSS + T cells + GM-CSF
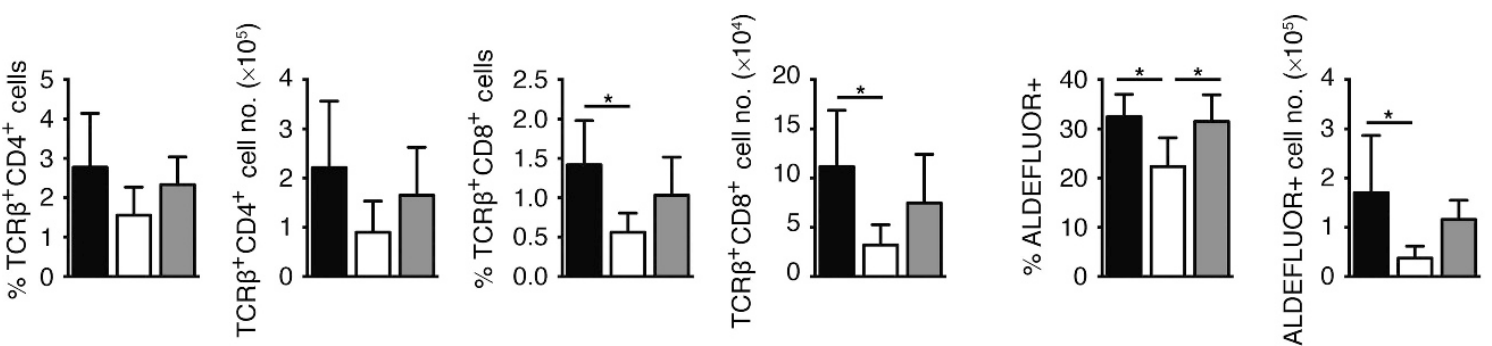
$\mathrm{CD} 45^{+1+} \mathrm{CD}^{+}$and $\mathrm{CD} 8{ }^{+} \mathrm{T}$ cells in $\mathrm{CD} 45 \mathrm{RAG}^{-1-}$ mice had significantly reduced frequency and number of $\alpha_{4} \beta_{7}$-expressing $\mathrm{T}$ cells (Figure 7a-e). Furthermore, there was also a reduced frequency of $\mathrm{CCR} 9^{+} \mathrm{CD} 8{ }^{+} \mathrm{T}$ cells (Figure 7a,e). CCR9 (C-C motif chemokine receptor 9) is a chemokine receptor known to facilitate homing to the small intestine. ${ }^{33,34}$ These results support the notion that the reduced numbers of $\mathrm{CD} 45^{+1+}$ T-cell effectors in the LP of CD45RAG ${ }^{-1-}$ mice after DSS-induced colitis is due to reduced imprinting of $\alpha_{4} \beta_{7}$, attributed to reduced numbers of RA-producing DCs.

\section{Exogenous GM-CSF and RA restores intestinal effector $\mathrm{CD}^{+1+} \mathrm{T}^{+/}$cells and promotes inflammation in CD45RAG $^{-1-}$ mice}

To test whether the reduced RA was leading to the decreased T-cell homing and gut inflammation in CD45RAG ${ }^{-1-}$ mice, we injected polyclonal CD45 ${ }^{+/+} \mathrm{T}$ cells and induced DSS colitis and then injected all-trans RA every other day from day 1 of the experiment, as had been carried out previously. ${ }^{22}$ Providing exogenous RA to CD45RAG ${ }^{-1-}$ mice significantly increased their weight loss and decreased colon lengths such that they were now similar to the $\mathrm{RAG}^{-1-}$ mice and distinct from the CD45RAG ${ }^{-1-}$ mice injected with vehicle only (Figure $7 f$ ). This demonstrates that exogenously added RA can rescue the CD45RAG ${ }^{-1-}$ defect and worsen the symptoms of DSS-induced colitis, in line with an emerging inflammatory role for RA. ${ }^{32}$

GM-CSF production by ILCs and intestinal stromal cells has been shown to induce the production of RA by intestinal DCs. ${ }^{25,35}$ As there were less GM-CSF producing Lin ${ }^{-}$Thy $1.2^{+}$ NK1.1 ${ }^{+}$ILCs in the colon of CD45RAG ${ }^{-1-}$ mice after DSS treatment, we next assessed whether exogenous GM-CSF could restore RA and T-cell recruitment to the colon of CD45RAG ${ }^{-1-}$ mice. Again, upon the injection of polyclonal $\mathrm{CD} 45^{+1+} \mathrm{T}$ cells and induction of DSS colitis, we injected recombinant GM-CSF every other day from day 1 of the experiment. Similar to the RA injections, exogenous GM-CSF significantly increased the weight loss of CD45RAG ${ }^{-1-}$ mice to the levels observed in the $\mathrm{RAG}^{-/-}$mice (Figure $7 \mathrm{~g}$ ). Furthermore, the injection of GM-CSF into CD45RAG ${ }^{-1-}$ mice increased the frequency and number of $\mathrm{T}$ cells in the colon toward the levels observed in the RAG ${ }^{-1-}$ mice (Figure $7 \mathbf{h}$ ). Finally, the exogenous GM-CSF significantly increased the percentage of ALDEFLUOR ${ }^{+} \mathrm{CD}_{103}{ }^{+}$DCs in the mLNs of CD45RAG ${ }^{-1-}$ mice to levels observed in the $\mathrm{RAG}^{-1-}$ mice (Figure 7i). This demonstrates the importance of GM-CSF in enhancing RA production capacity and effector T-cell homing to the intestine upon DSS-induced colitis and suggests a positive role for CD45 in the production of GM-CSF and RA by innate immune cells.

\section{DISCUSSION}

Here we show a novel role for $\mathrm{CD} 45$ in regulating both adaptive and innate immune cell functions in intestinal inflammation. In the DSS model of colitis, equal frequencies of $\mathrm{T}$ cells were present in the colon and small intestine of WT and CD $45^{-1-}$ mice which was surprising given the reduced frequency of $\mathrm{T}$ cells in the $\mathrm{CD} 45^{-l-}$ mice compared with WT mice prior to colitis, and given the reduced frequencies of $\mathrm{T}$ cells in the spleen, $\mathrm{mLNs}$, and lung both before and after colitis. This prevalence of $\mathrm{T}$ cells in the inflamed gut of $\mathrm{CD} 45^{-1-}$ mice was attributed to the increased expression of the gut-homing molecule $\alpha 4 \beta 7$ on the CD $45^{-1-}$ T cells, which was observed in the mLNs both before and after colitis. This was thought to be an intrinsic defect in these cells as RA, the molecule responsible for this imprinting, was not increased in CD $45^{-1-}$ DCs in the mLNs, and because, when $\mathrm{CD} 45^{+1+} \mathrm{T}$ cells were transferred into CD45RAG ${ }^{-1-}$ mice and DSS colitis was induced, these $\mathrm{T}$ cells did not show increased $\alpha 4 \beta 7$ expression. This suggests that the absence of $\mathrm{CD} 45$ on $\mathrm{T}$ cells results in the upregulation of the gut-homing molecule $\alpha 4 \beta 7$. The loss of CD 45 on T cells has also been reported to increase the expression of another integrin, LFA $-1,{ }^{8}$ and modulate integrin-mediated T-cell adhesion. ${ }^{29}$ The $\mathrm{CD} 45^{-1-} \mathrm{T}$ cells had increased expression of the guthoming molecule, $\alpha 4 \beta 7$, and substantial T-cell numbers in the colon, despite a reduced percentage of ALDEFLUOR-expressing CD $45^{-1-}$ DCs in the mLNs of CD $45^{-1-}$ mice in DSS colitis, indicative of reduced RA production. One possible explanation for this is that the absence of CD45 on T cells may lead to the upregulation of $\alpha 4 \beta 7$ expression, to help compensate for the reduced RA produced by the innate immune cells.

More proinflammatory cytokine-producing $\mathrm{CD} 45^{-1-}$ cells ( $\mathrm{T}$ cells, ILCs, and DCs) were present in the colon of CD45 ${ }^{-1-}$ mice compared with WT mice with DSS-induced colitis, signifying a negative regulatory effect of CD45 on proinflammatory cytokine production in these cells. However, when we analyzed cytokine production in the colon of CD45RAG ${ }^{-1-}$ mice after DSS-induced colitis, we found that, in the absence of an adaptive immune system, ILCs made less proinflammatory mediators. In the $\mathrm{CD} 45^{-1-}$ mice, the $\mathrm{CD} 45^{-1-} \mathrm{T}$ cells made more GM-CSF than WT $\mathrm{T}$ cells in the inflamed intestine that may be sufficient to overcome an initial defect in GM-CSF production by the ILCs and provide a positive feedback loop that promotes myeloid cell proliferation and RA production, which in turn feeds back and amplifies GM-CSF production by ILCs, as has been suggested for DCs and stromal cells. ${ }^{33}$

In the absence of $\mathrm{T}$ or $\mathrm{B}$ cells in the CD45RAG ${ }^{-1-}$ mice, $\mathrm{CD} 45^{-1-}$ ILCs from the LP produced less IFN $\gamma$, GM-CSF, and IL-22 in DSS colitis. As GM-CSF can promote intestinal myeloid survival, ${ }^{21}$ it is possible that reduced GM-CSF may account for the reduced numbers of myeloid cells observed in the inflamed colon of CD45RAG ${ }^{-1-}$ mice. Reduced GM-CSF levels may also contribute to the reduced capacity of DCs to produce RA in the colon in DSS-induced colitis, as addition of exogenous GM-CSF rescued RA production by DCs. RA may feedback on ILCs as one report shows that RA promotes IL-22 production by ILCs, including $\gamma \delta \mathrm{T}$ cells, ${ }^{22}$ which has a protective role in the gut $^{24,36,37}$ and protects mice from DSS colitis. ${ }^{22}$ Thus the reduced levels of RA and IL-22 in the CD45RAG ${ }^{-1-}$ mice may contribute to the increased intestinal inflammation observed in the CD45RAG ${ }^{-1-}$ mice upon 
DSS-induced colitis. When CD $45^{+1+} \mathrm{T}$ cells were injected into CD45RAG ${ }^{-1-}$ mice and DSS colitis induced, in contrast to the situation in the $\mathrm{CD} 45^{-1-}$ mice, there were reduced numbers of effector $\mathrm{T}$ cells in the intestine and reduced inflammation as assessed by cell infiltration in the LP, increased colon length, and decreased weight loss. In contrast to the $\mathrm{CD} 45^{-1-} \mathrm{T}$ cells, the WT T cells transferred into CD45RAG ${ }^{-1-}$ mice had significantly less $\alpha 4 \beta 7^{+} \mathrm{T}$ cells and lower $\alpha 4 \beta 7^{+}$expression levels in the mLNs after DSS-induced colitis compared with WT $\mathrm{T}$ cells injected into $\mathrm{RAG}^{-1-}$ mice. This reduced induction of $\alpha 4 \beta 7$ and reduced T-cell homing to the intestine was attributed to the reduced capacity of DCs to produce RA as the addition of exogenous RA increased inflammation, as assessed by decreased colon length and increased weight loss. This was further supported by the addition of GM-CSF, which increased the percentage of RAproducing DCs and led to increased $\mathrm{T}$ cells in the colon. This implies a positive effect of CD45 on the production of RA by DCs, which in turn affects the expression of $\alpha 4 \beta 7$ and the homing of T cells to the gut. ${ }^{27}$ As GM-CSF can rescue the RA defect in the CD45 ${ }^{-1-}$ DCs, this may be an extrinsic effect of CD45 deficiency.

GM-CSF production by ILCs and stromal cells maintains the macrophage and DC populations in the gut at homeostasis and promotes RA production by DCs. ${ }^{25,35}$ As we do not see a reduced myeloid population in the colon prior to colitis (data not shown), CD45 may be important for inflammationinduced GM-CSF levels in the gut. We show that, upon DSS colitis, exogenous GM-CSF restores RA production by $\mathrm{CD}_{4} 5^{-1-} \mathrm{CD}_{103}{ }^{+}$DCs in CD45RAG ${ }^{-1-}$ mice, but unlike homeostasis, where Treg conversion and homing to the gut predominates, here we see enhanced effector T-cell homing to the inflamed gut. Whether CD45 functions intrinsically and/or extrinsically to modulate GM-CSF and IL-22 production by $\mathrm{Lin}^{-}$Thy $1.2^{+} \mathrm{NK} 1.1^{+}$ILCs is not known. It is possible that the lack of CD45 in myeloid cells may contribute by limiting the initial signals to the ILCs, for example, by limiting IL- $1 \beta$ production that has been shown to regulate GM-CSF production by ILCs. ${ }^{25}$ It is also possible that CD45 deficiency on ILCs regulates their GM-CSF and IL-22 production. The tyrosine kinase, Lyn, has been shown to be important for regulating IL-22 production by ILCs in DSS colitis ${ }^{36}$ and as $\mathrm{CD} 45$ is a regulator of Lyn, ${ }^{38}$ it may regulate IL-22 and GM-CSF production by regulating Lyn.

GM-CSF has previously been shown to stimulate DCs to acquire RA-producing capacity. ${ }^{39} \mathrm{GM}^{-} \mathrm{CSF}^{-1-}$ mice have reduced myeloid cells in the intestine with reduced capacity to make RA, and these mice were unable to induce oral tolerance as a result of insufficient conversion of naive $\mathrm{T}$ cells to Tregs. ${ }^{25}$ In DSS colitis, CD45RAG ${ }^{-1-}$ mice have a similar phenotype to that of GM-CSF${ }^{-1-}$ mice in that they have reduced myeloid cells in the inflamed gut and a lower capacity to produce RA by the myeloid cells locally in the intestine and mLNs. After the adoptive transfer of $\mathrm{CD} 45^{+1+} \mathrm{T}$ cells, there were less (not more) Tregs in the inflamed colon and significantly reduced numbers of effector $\mathrm{CD} 4$ and $\mathrm{CD} 8 \mathrm{~T}$ cells in the
CD45RAG ${ }^{-1-}$ mice compared with the RAG ${ }^{-1-}$ mice, which led to reduced DSS-induced inflammation. Although RA is known to promote Treg conversion at homeostasis and induce gut tropic molecules on Tregs, ${ }^{19}$ here we show that GM-CSF increases RA production capacity and helps direct WT effector T cells to the gut in DSS colitis. Consistent with this, one report showed that RA production is necessary for expression of gut-homing receptors on effector $\mathrm{CD} 4{ }^{+} \mathrm{T}$ cells, and when WT mice were given a vitamin A-deficient diet and infected with a parasite, Toxoplasma gondii, or when mice deficient for RA receptor $\alpha$ were infected with this parasite, there was insufficient effector $\mathrm{CD} 4{ }^{+} \mathrm{T}$ cells in the intestine and inefficient clearance of the parasite. ${ }^{32}$

Overall, this study demonstrates an inflammatory role for GM-CSF in DSS-induced colitis by enhancing the percentage of RA-producing DCs and gut-tropic effector $\mathrm{T}$ cells in a mouse model of colitis. We identify differing roles for the phosphatase $\mathrm{CD} 45$ in innate and adaptive immune cells in intestinal inflammation. CD45 is required for optimal GM-CSF and RA production in innate immune cells that affects $\alpha 4 \beta 7$ expression and the homing of effector $\mathrm{T}$ cells to the intestine. Conversely, the absence of CD45 on adaptive immune cells leads to enhanced $\alpha 4 \beta 7$ expression on T cells and homing to the intestine. Proinflammatory cytokine production is increased in these T cells in DSS-induced colitis that may create a positive feedback loop involving both DCs and ILCs.

\section{METHODS}

Mice. C57BL/6, CD45 ${ }^{-/-}$Exon $9,{ }^{4}$ and $\mathrm{RAG} 1^{-/-}$mice were obtained from The Jackson Laboratory (Sacramento, CA). CD45 ${ }^{-1-}$ Exon 6 mice $^{5}$ were obtained from J. Penninger. CD45E6 ${ }^{-1-}$ and CD45E $9^{-1-}$ mice were backcrossed for six to nine generations, respectively, onto the C57BL/6 background. CD45E9RAG ${ }^{-1-}$ and CD45E6RAG ${ }^{-1-}$ mice were generated by crossing $\mathrm{CD} 45^{-1-}$ mice with RAG1 ${ }^{-1-}$ mice to homozygosity. Mice were also re-derived and bred under specific pathogen-free conditions at the Centre for Disease Modeling Animal Facility at the University of British Columbia. The mice were maintained and used for experimentation in accordance with the Canadian Council of Animal Care Guidelines and with approval from the University of British Columbia Animal Care Committee. Mice were used between 6 and 14 weeks of age and matched for age and gender.

Induction of colitis, cell transfers, and injection of all-trans RA and GM-CSF. Experimental colitis was induced by dissolving 3\% (w/v) dextran sodium sulphate (molecular mass: 36,000-50,000 from MP Biomedicals, Santa Ana, CA) into drinking water and given to mice for 7 days. Normal drinking water was then given for an additional 1-2 days. The animals were weighed daily and assessed for clinical signs of colitis such as rectal bleeding. Polyclonal $\mathrm{T}$ cells were isolated from $\mathrm{C} 57 \mathrm{Bl} / 6$ spleens and lymph nodes (mesenteric, auxiliary, and inguinal) by negative depletion after labeling cells with biotinylated antibodies, CD11c (N418), Ter119 (TER119), CD11b (M1/70), and B220 (RA36B2) and then adding antibiotin beads (Miltenyi Biotec, Auburn, CA) to pass through magnetic columns (Miltenyi Biotec) for collection of the unlabeled fraction. T cells were at least 93\% pure and $3-5 \times 10^{6} \mathrm{~T}$ cells/mouse were injected once intravenously on day 0 of a DSS experiment. Where indicated, $200 \mu \mathrm{g}$ per mouse of all-trans RA (Enzo Life Sciences, Farmingdale, NY), $5 \mu \mathrm{g}$ per mouse recombinant mouse GM-CSF (BioLegend, San Diego, CA), phosphate-buffered saline, or 
vehicle (a mixture of 16\% dimethyl sulfoxide and peanut oil (Sigma, Oakville, ON, Canada)) were injected intraperitoneally every other day from day 1 of a DSS experiment.

Histology. Naive mice and mice on days 7-9 of DSS were killed, the colons removed, and a portion of the distal colon was fixed in $10 \%$ formalin. After paraffin embedding, 5- $\mu \mathrm{m}$-thick cross-sections were cut and stained with hematoxylin and eosin (Wax-It, Vancouver, British Columbia, Canada). Sections were scored as previously described. ${ }^{40}$ Briefly, they were given a score of 0 (none), 1,2 , or 3 (severe) and assessed for colon damage for the following parameters: degree of leukocyte infiltration, crypt architecture damage, and muscle thickening. Sections were also scored with a 0 for the presence and 1 for the absence of goblet cells and crypt abscesses. All scores were assessed blindly and summed to get a total score.

Cell isolation. Single-cell suspensions from the mLNs and spleens were isolated by incubating minced organs with $1 \mathrm{mg} \mathrm{ml}^{-1}$ of collagenase IV (Worthington, Lakewood, NJ) for $20 \mathrm{~min}$ and passing them through 70$\mu \mathrm{m}$ strainers. For the spleens, red blood cells were lysed with $0.84 \%$ ammonium chloride and $2 \mathrm{~mm}$ Tris- $\mathrm{HCl} \mathrm{pH} 7.2$ at room temperature for 5 min. Intestinal LP cells were isolated from the large intestine. In brief, colon from the cecum to the rectum was dissected from killed mice and opened longitudinally and then cut into approximately $0.5-\mathrm{cm}$ pieces and incubated for $20-30 \mathrm{~min}$ at $37^{\circ} \mathrm{C}$ in a solution of phosphate-buffered saline, 5\% fetal calf serum, and $4 \mathrm{~mm}$ EDTA for at least three times to remove epithelial cells. Colon pieces were then washed twice with a solution of phosphate-buffered saline and 5\% fetal calf serum to remove the EDTA and then minced with a scalpel, incubated twice at $37^{\circ} \mathrm{C}$ for $40 \mathrm{~min}$ in a solution of RPMI, $5 \%$ fetal calf serum, and $1 \mathrm{mg} \mathrm{ml}^{-1}$ of collagenase IV and passed through a $70-\mu \mathrm{m}$ strainer to obtain the LP cells. $^{41}$

Intracellular staining and flow cytometry. Single-cell suspensions were blocked for FcR binding with $2.4 \mathrm{G} 2$ cell culture supernatant and then labeled with direct fluorochrome conjugates for the surface markers CD11c (N418), CD11b (M1/70), CD103 (2E7), MHCII (I-A/I-E), Gr1 (RB6-8C5), NK1.1 (PK136), CCR9 (CW-1.2), $\alpha 4 \beta 7$ (DATk32), TCR $\beta$ (H57-597), CD8 $\alpha$ (53-6.7), and CD4 (GK1.5) from eBioscience (San Diego, CA). Thy1.2 (30-H12), CD19 (1D3), and CD3 (2C11) were from the Biomedical Research Centre Antibody Facility (Vancouver, BC, Canada). Isolated cells from the spleen and LP were stimulated with phorbol myristate acetate $\left(50 \mathrm{ng} \mathrm{ml}^{-1}\right)$ and ionomycin $\left(500 \mathrm{ng} \mathrm{ml}^{-1}\right)$ for $4-6 \mathrm{~h}$ in the presence of Brefeldin A $\left(10 \mu \mathrm{g} \mathrm{ml}^{-1}\right)$, which was added at the same time, for the assessment of cytokine production. For the analysis of ILCs, CD11b (M1/70), Gr1 (RB6-8C5), CD11c (N418), CD3 (2C11), and CD19 (1D3) were used in the lineage mix and the ILCs were selected for by Thy $1.2^{+}$and in some cases the NK $1.1^{+}$subset of the Thy $1.2^{+}$ILC population was examined. Intracellular labeling was performed with the Foxp3 fixation/ permeabilization concentrate and associated buffers (eBioscience) for IL-22 (1H8PWSR), IFN $\gamma$ (XMG1.2), IL-17A (ebio17B7), ROR $\gamma \mathrm{t}$ (B2D), Foxp3 (FJK-16s), and TNF $\alpha$ (MP6-XT22) from eBioscience and GM-CSF (MP1-22E9) from BioLegend. Samples were analyzed on a FACSCanto or LSRII (BD, San Jose, CA) with the FlowJo software (Tree Star, Ashland, OR) with isotype controls to determine gating and exclude non-specific binding. The presence of aldehyde dehydrogenase activity in cells was determined using the ALDEFLUOR Staining Kit (STEMCELL Technologies, Vancouver, BC, Canada) as per the manufacturer's instructions. All cells were labeled with a viability dye, either 4,6-diamidino-2-phenylindole or 7-aminoactinomycin D when labeling surface markers or with a fixable/viability dye (Invitrogen, Burlington, ON, Canada) when labeling intracellular proteins.

Statistical analysis. Statistical analyses were performed using the Graphpad Prism software (La Jolla, CA). Data were analyzed using the unpaired Student's $t$-test. For multiple analyses, analysis of variance with repeated measures was used, and where significance was found,
Bonferroni multiple comparisons test was used for two-way and Dunnett's test was used for one-way analysis of variance for identifying differences between groups. When $P<0.05$, the statistical difference was considered significant.

SUPPLEMENTARY MATERIAL is linked to the online version of the paper at http://www.nature.com/mi

\section{ACKNOWLEDGMENTS}

We thank the UBC Flow Cytometry Facility. This work was supported by a grant from the Canadian Institutes of Health Research (no. MOP-77712). M.C. was supported by a summer studentship from the Natural Sciences and Engineering Council of Canada. A.S. was supported by a Canadian Institutes of Health Research-Banting and Best Master's studentship and, in partnership with the Canadian Digestive Health Foundation, a Doctoral studentship.

\section{DISCLOSURE}

The authors declared no conflict of interest.

(c) 2016 Society for Mucosal Immunology

\section{REFERENCES}

1. Cross, J.L., Kott, K., Miletic, T. \& Johnson, P. CD45 regulates TLR-induced proinflammatory cytokine and IFN-beta secretion in dendritic cells. J. Immunol. 180, 8020-8029 (2008).

2. Piercy, J., Petrova, S., Tchilian, E.Z. \& Beverley, P.C. CD45 negatively regulates tumour necrosis factor and interleukin- 6 production in dendritic cells. Immunology 118, 250-256 (2006).

3. Irie-Sasaki, J. et al. CD45 is a JAK phosphatase and negatively regulates cytokine receptor signalling. Nature 409, 349-354 (2001).

4. Byth, K.F. et al. CD45-null transgenic mice reveal a positive regulatory role for CD45 in early thymocyte development, in the selection of CD4 + CD8 + thymocytes, and B cell maturation. J. Exp. Med. 183, 1707-1718 (1996).

5. Kishihara, K. et al. Normal B lymphocyte development but impaired T cell maturation in CD45-exon6 protein tyrosine phosphatase-deficient mice. Cell 74, 143-156 (1993)

6. Mee, P.J., Turner, M., Basson, M.A., Costello, P.S., Zamoyska, R. \& Tybulewicz, V.L. Greatly reduced efficiency of both positive and negative selection of thymocytes in CD45 tyrosine phosphatase-deficient mice. Eur. J. Immunol. 29, 2923-2933 (1999).

7. Saunders, A.E., Shim, Y.A. \& Johnson, P. Innate immune cell CD45 regulates lymphopenia-induced T cell proliferation. J. Immunol. 193, 2831-2842 (2014).

8. Kong, Y.Y., Kishihara, K., Yoshida, H., Mak, T.W. \& Nomoto, K. Generation of $T$ cells with differential responses to alloantigens in CD45 exon 6-deficient mice. J. Immunol. 154, 5725-5735 (1995).

9. Lopez, M.C. \& Holmes, N. Phenotypical and functional alterations in the mucosal immune system of CD45 exon $9 \mathrm{KO}$ mice. Int. Immunol. 17, 15-25 (2005).

10. Saleh, M. \& Trinchieri, G. Innate immune mechanisms of colitis and colitisassociated colorectal cancer. Nat. Rev. Immunol. 11, 9-20 (2011).

11. Kim, T.W. et al. Involvement of lymphocytes in dextran sulfate sodiuminduced experimental colitis. World J. Gastroenterol. 12, 302-305 (2006).

12. Morgan, M.E. et al. New perspective on dextran sodium sulfate colitis: antigen-specific T cell development during intestinal inflammation. PLOS One 8, e69936 (2013).

13. Denning, T.L., Wang, Y.C., Patel, S.R., Williams, I.R. \& Pulendran, B. Lamina propria macrophages and dendritic cells differentially induce regulatory and interleukin 17-producing Tcell responses. Nat. Immunol. 8, 1086-1094 (2007)

14. Rivollier, A., He, J., Kole, A., Valatas, V. \& Kelsall, B.L. Inflammation switches the differentiation program of Ly6Chi monocytes from antiinflammatory macrophages to inflammatory dendritic cells in the colon. J. Exp. Med. 209, 139-155 (2012).

15. Zigmond, E. et al. Macrophage-restricted interleukin-10 receptor deficiency, but not IL-10 deficiency, causes severe spontaneous colitis. Immunity 40, 720-733 (2014). 
16. Schulz, O. et al. Intestinal CD103 +, but not CX3CR1 +, antigen sampling cells migrate in lymph and serve classical dendritic cell functions. J. Exp. Med. 206, 3101-3114 (2009).

17. Worbs, T. et al. Oral tolerance originates in the intestinal immune system and relies on antigen carriage by dendritic cells. J. Exp. Med. 203, 519-527 (2006).

18. Annacker, O. etal. Essential role for CD103 in the Tcell-mediated regulation of experimental colitis. J. Exp. Med. 202, 1051-1061 (2005).

19. Coombes, J.L. et al. A functionally specialized population of mucosal CD103 + DCs induces Foxp3 + regulatory T cells via a TGF-beta and retinoic acid-dependent mechanism. J. Exp. Med. 204, 1757-1764 (2007).

20. Sun, C.M. et al. Small intestine lamina propria dendritic cells promote de novo generation of Foxp3 T reg cells via retinoic acid. J. Exp. Med. 204, 1775-1785 (2007).

21. Buonocore, S. et al. Innate lymphoid cells drive interleukin-23-dependent innate intestinal pathology. Nature 464, 1371-1375 (2010).

22. Mielke, L.A. et al. Retinoic acid expression associates with enhanced IL-22 production by gammadelta Tcells and innate lymphoid cells and attenuation of intestinal inflammation. J. Exp. Med. 210, 1117-1124 (2013).

23. Eken, A., Singh, A.K., Treuting, P.M. \& Oukka, M. IL-23R + innate lymphoid cells induce colitis via interleukin-22-dependent mechanism. Mucosal Immunol. 7, 143-154 (2014).

24. Cox, J.H. et al. Opposing consequences of IL-23 signaling mediated by innate and adaptive cells in chemically induced colitis in mice. Mucosal Immunol. 5, 99-109 (2012).

25. Mortha, A. et al. Microbiota-dependent crosstalk between macrophages and ILC3 promotes intestinal homeostasis. Science 343, 1249288 (2014).

26. Longman, R.S. et al. CX(3)CR1(+) mononuclear phagocytes support colitis-associated innate lymphoid cell production of IL-22. J. Exp. Med. 211, 1571-1583 (2014).

27. Gorfu, G., Rivera-Nieves, J. \& Ley, K. Role of beta7 integrins in intestinal lymphocyte homing and retention. Curr. Mol. Med. 9, 836-850 (2009).

28. Wagner, N. et al. Critical role for beta7 integrins in formation of the gutassociated lymphoid tissue. Nature 382, 366-370 (1996).

29. Shenoi, H., Seavitt, J., Zheleznyak, A., Thomas, M.L. \& Brown, E.J. Regulation of integrin-mediated $\mathrm{T}$ cell adhesion by the transmembrane protein tyrosine phosphatase CD45. J. Immunol. 162, 7120-7127 (1999).
30. Wong, N.K., Lai, J.C., Birkenhead, D., Shaw, A.S. \& Johnson, P. CD45 down-regulates Lck-mediated CD44 signaling and modulates actin rearrangement in T cells. J. Immunol. 181, 7033-7043 (2008).

31. Brown, C.C. et al. Retinoic acid is essential for Th1 cell lineage stability and prevents transition to a Th17 cell program. Immunity $\mathbf{4 2 ,}$ 499-511 (2015).

32. Hall, J.A. et al. Essential role for retinoic acid in the promotion of CD4(+) $\mathrm{T}$ cell effector responses via retinoic acid receptor alpha. Immunity 34 , 435-447 (2011).

33. Kunkel, E.J. et al. Lymphocyte CC chemokine receptor 9 and epithelial thymus-expressed chemokine (TECK) expression distinguish the small intestinal immune compartment: epithelial expression of tissue-specific chemokines as an organizing principle in regional immunity. J. Exp. Med. 192, 761-768 (2000).

34. Svensson, M. et al. CCL25 mediates the localization of recently activated CD8alphabeta $(+)$ lymphocytes to the small-intestinal mucosa. J. Clin. Invest. 110, 1113-1121 (2002).

35. Vicente-Suarez, I. et al. Unique lamina propria stromal cells imprint the functional phenotype of mucosal dendritic cells. Mucosal Immunol. 8 , 141-151 (2015).

36. Bishop, J.L. et al. Lyn activity protects mice from DSS colitis and regulates the production of IL-22 from innate lymphoid cells. Mucosal Immunol. 7 , 405-416 (2014).

37. Zenewicz, L.A., Yancopoulos, G.D., Valenzuela, D.M., Murphy, A.J., Stevens, S. \& Flavell, R.A. Innate and adaptive interleukin-22 protects mice from inflammatory bowel disease. Immunity 29, 947-957 (2008).

38. Johnson, P., Samarakoon, A., Saunders, A. \& Harder, K. CD45 (PTPRC). Encyclopedia of Signaling Molecules, 328-334 ((Springer2012)).

39. Yokota, A. et al. GM-CSF and IL-4 synergistically trigger dendritic cells to acquire retinoic acid-producing capacity. Int. Immunol. 21, 361-377 (2009).

40. Kim, J.J., Shajib, M.S., Manocha, M.M. \& Khan, W.I. Investigating intestinal inflammation in DSS-induced model of IBD. J. Vis. Exp. 60, 3678 (2012).

41. Uhlig, H.H. et al. Characterization of Foxp3 + CD4 + CD25 + and IL-10secreting CD4 + CD25 + T cells during cure of colitis. J. Immunol. 177, 5852-5860 (2006). 